NBER WORKING PAPER SERIES

\title{
LEARNING ABOUT THE NEIGHBORHOOD
}

\author{
Zhenyu Gao \\ Michael Sockin \\ Wei Xiong
}

Working Paper 26907

http://www.nber.org/papers/w26907

\author{
NATIONAL BUREAU OF ECONOMIC RESEARCH \\ 1050 Massachusetts Avenue \\ Cambridge, MA 02138 \\ March 2020
}

We are grateful to Itay Goldstein, Laura Veldkamp and seminar participants of 2018 AEA Meetings, 2018 NBER Asset Pricing Meeting, Fordham University, McGill University and UC Berkeley for helpful comments. The views expressed herein are those of the authors and do not necessarily reflect the views of the National Bureau of Economic Research.

NBER working papers are circulated for discussion and comment purposes. They have not been peer-reviewed or been subject to the review by the NBER Board of Directors that accompanies official NBER publications.

(C) 2020 by Zhenyu Gao, Michael Sockin, and Wei Xiong. All rights reserved. Short sections of text, not to exceed two paragraphs, may be quoted without explicit permission provided that full credit, including $\odot$ notice, is given to the source. 
Learning about the Neighborhood

Zhenyu Gao, Michael Sockin, and Wei Xiong

NBER Working Paper No. 26907

March 2020

JEL No. E22,E44,G1,R3,R31

\section{ABSTRACT}

We develop a model to analyze information aggregation and learning in housing markets. In the presence of pervasive informational frictions, housing prices serve as important signals to households and capital producers about the economic strength of a neighborhood. Our model provides a novel mechanism for amplification through learning in which noise from the housing market can propagate to the local economy, distorting not only migration into the neighborhood, but also the supply of capital and labor. We provide consistent evidence of our model implications for housing price volatility and new construction using data from the recent U.S. housing cycle.

Zhenyu Gao

Chinese University of Hong Kong

Hong Kong

gaozhenyu@baf.cuhk.edu.hk

Michael Sockin

Department of Finance

UT Austin McCombs School of Business

Austin, TX 78712

michael.sockin@mccombs.utexas.edu

\author{
Wei Xiong \\ Princeton University \\ Department of Economics \\ Bendheim Center for Finance \\ Princeton, NJ 08450 \\ and NBER \\ wxiong@princeton.edu
}


Economists have long been puzzled by the substantial price fluctuations experienced in housing markets. The recent U.S. housing cycle in the 2000s has renewed attention on this important issue as it has proved difficult to provide fundamental-based explanations for housing boom-bust cycles both in the aggregate and across regions (Glaeser, Gyourko, Morales, and Nathanson 2014, Glaeser and Nathanson 2015). While a growing literature has emphasized the role of expectations, such as extrapolative beliefs, in shaping housing dynamics, e.g., Case and Shiller (2003), and Glaeser, Gyourko, and Saiz (2008), it remains a challenge to link the formation of these expectations to the excessive volatility in housing prices observed in recent housing boom and bust cycles. ${ }^{1}$

In this paper, we address this challenge by developing a model to analyze how informational frictions affect the learning and beliefs of households and capital producers about a neighborhood, which, in turn, influence both local housing markets and investment decisions. We extend the coordination problem with dispersed information to investigate its role in amplifying the agglomeration effects that underpin the formation of neighborhoods and cities. In contrast to conventional models of learning, in which noise often dampens real activity, our model provides a novel amplification mechanism in which learning can amplify and propagate noise in housing markets and local real investment. In addition, it is able to generate rich non-monotonic patterns in housing cycles with respect to supply elasticity, as well as the degree of local consumption complementarity, another dimension in which neighborhoods differ.

Our model features a continuum of households, each of which has the choice of whether to move into an open neighborhood, which can be viewed as a Metropolitan Statistical Area (MSA) or city, by buying a house. To capture the idea that productive households prefer to live with other productive households, we assume that each household has a Cobb-Douglas utility function over consumption of its own good and goods produced by other households in the neighborhood. This complementarity in households' consumption motivates each household to learn about an unobservable economic strength of the neighborhood, which determines the common productivity of all households and which leads to complementarity in their housing demand. To produce its good, each household requires both labor, which

\footnotetext{
${ }^{1}$ Intuitively, by amplifying housing price fluctuations, extrapolation makes housing cycles monotonic with respect to the supply elasticity of land. This prediction, however, does not fully capture the cross-section of the recent U.S. housing cycle. Many researchers, including Glaeser (2013), Davidoff (2013), and Nathanson and Zwick (2018), have noted that the housing price boom and bust were most pronounced in areas that were not particularly constrained by the supply of land, including Las Vegas and Phoenix.
} 
it supplies, and local capital, such as office space and warehouse. Since the price of local capital depends on its marginal product across households in the neighborhood, competitive capital producers must also form expectations about the neighborhood's economic strength when determining how much local capital to develop, providing a channel to amplify the economic effects of housing market noise.

Although previously unexplored in the housing literature, it is intuitive that local housing markets provide a useful platform for aggregating private information about the economic strength of a neighborhood. The traded housing price reflects the net effect of demand and supply-side factors, in a similar spirit to the classic models of Grossman and Stiglitz (1980) and Hellwig (1980) for information aggregation in asset markets. In contrast to conventional security markets, in which who owns the asset does not affect asset cash flows, which households own houses determines the convenience yield of living in the neighborhood, through the quality of services and social interactions the neighborhood provides, which, in turn, determines the value of housing. Which households buy houses also guides the investment decisions of capital producers, who must predict future neighborhood demographics when deciding how much capital to supply. As a result of the complementarity and informational frictions, noise in the housing market can impact the local economy because households and capital producers use housing market signals when forecasting each other's housing and real investment decisions. This gives rise to a feedback loop, through which the extrapolative-like behavior of households and capital producers, induced by learning, leads to not only a more pronounced housing cycle but also an oversupply of new housing and local capital, consistent with the empirical findings of Gao, Sockin, and Xiong (2019). Through this feedback loop, learning can amplify housing price movements and contribute to excessive price volatility.

Our analysis illustrates how the transmission of noise in housing markets to real estate and production outcomes varies across different neighborhoods by the elasticity of local housing supply - in a hump-shaped pattern. At intermediate supply elasticities, the housing price has balanced weights on the demand-side and supply-side fundamentals. In the presence of informational frictions, the balanced weights make learning from the housing price particularly noisy. In contrast, at one extreme when housing supply is infinitely inelastic, the housing price is fully determined by housing demand, and perfectly reveals the strength of the neighborhood; at the other extreme, when housing supply is perfectly elastic, housing prices are fully determined by housing supply. At both extremes, learning does not distort 
the housing price. As a result, the noise effects induced by informational frictions on housing prices are strongest at intermediate supply elasticities.

Our analysis also examines this transmission across the degree of households' consumption complementarity, which one can interpret as the share of consumption from local nontradable industries. The distortionary effects of learning on the housing market tend to increase with complementarity, since greater complementarity makes learning about the neighborhood strength a more important part of each household's decisions. As such, our analysis predicts a monotonically increasing pattern in the magnitudes of housing price boom and bust with respect to the degree of complementarity.

To illustrate empirical relevance of our model, we sort the cross-section of MSAs in the U.S. by their supply elasticity and the degree of complementarity. We systematically document that the non-monotonic pattern with respect to supply elasticity was more ubiquitous during the recent U.S. housing bubble than previously appreciated - in not only the magnitude of the housing price cycle, which serves as a proxy of the volatility amplification illustrated by our model, but also in new housing construction. Moreover, the magnitude of the housing price cycle appears to be monotonically increasing across the degree of complementarity. Taken together, these cross-sectional patterns of recent housing boom-bust cycles confirm our model implications, further validating the necessity for our new economic mechanism in which expectations interact with housing cycles beyond extrapolative beliefs.

Also different from the conventional linear equilibria in asset market models, each household's neighborhood selection makes our model inherently nonlinear. Nevertheless, we are able to derive the equilibrium analytically, building on and contributing to the growing literature that analyzes information aggregation in nonlinear settings. Goldstein, Ozdenoren, and Yuan (2013) investigate the feedback to the investment decisions of a single firm when managers, but not investors, learn from prices. Albagli, Hellwig, and Tsyvinski $(2015,2017)$ focus on the role of asymmetry in security payoffs in distorting asset prices and firm investment incentives when future shareholders learn from prices to determine their valuations. These models commonly employ risk-neutral agents, normally distributed asset fundamentals, and position limits to deliver tractable nonlinear equilibria. In contrast, we focus on the feedback induced by learning from housing prices to households' moving and consumption decisions and capital producers' investment decisions. By showing that the cutoff equilibrium framework can be adopted to analyze these richer learning effects, our model substantially expands 
the scope of this framework to a general equilibrium real business cycle environment. In this regard, our model also adds to the literature, e.g., Bond, Edmans and Goldstein (2012), on the real effects of learning from trading prices.

While long appreciated as important explanation for housing market behavior, such as in Garmaise and Moskowitz (2004), Kurlat and Stroebel (2014), Favara and Song (2014), and Bailey et al. (2017), informational frictions have yet to be applied to understanding the recent U.S. housing cycle and its real effects. The literature has instead focused on other causes ranging from credit expansion and fraudulent lending practices to speculation and optimistic, often extrapolative, expectations. ${ }^{2}$ By anchoring household expectations to local economic conditions, our theory provides guidance as to where optimism and overreaction had the most pronounced impact on housing and local economic outcomes during the boom, and offers novel empirical predictions on non-monotonic patterns in housing cycles and new construction with respect to supply elasticity and the degree of complementarity. In addition, our mechanism can rationalize the synchronized boom and bust cycles in commercial real estate markets, in which prices and new construction rose across the U.S. despite the bubble in housing (e.g., Gyourko (2009) and Levitin and Wachter (2013)). By impacting the demand curve for housing, informational frictions complement the credit expansion and fraud channels and, by facilitating heterogeneous beliefs, can give rise to speculative demand.

Our model adds to the literature on the theoretical modeling of housing cycles. Burnside, Eichenbaum, and Rebelo (2016) offer a model of housing market booms and busts based on the epidemic spreading of optimistic or pessimistic beliefs among home buyers through their social interactions. Nathanson and Zwick (2018) study the hoarding of land by home builders with heterogeneous beliefs in intermediate elastic areas as a mechanism to amplify price volatility in the recent U.S. housing cycle. Piazzesi and Schneider (2009) investigate how a small population of optimists can inflate housing prices by driving transaction volume. Glaeser and Nathanson (2017) presents a model of biased learning in housing markets in which the incorrect inference by home buyers gives rise to correlated errors in housing demand forecasts over time, which, in turn, generate excess volatility, momentum, and mean-reversion

\footnotetext{
${ }^{2}$ For credit expansion, see, for instance, Mian and Sufi (2009, 2011) and Albanesi et al. (2017). For fraudulent lending practices, see Keys et al. (2009) and Griffin and Maturana (2015). For speculation, see Chinco and Mayer (2015), Nathanson and Zwick (2018), DeFusco, Nathanson, and Zwick (2017), and Gao, Sockin and Xiong (2019). For extrapolative expectations, see Case and Shiller (2003), Glaeser, Gyourko, and Saiz (2008), Piazzesi and Schneider (2009), Cheng, Raina and Xiong (2014), and Glaeser and Nathanson (2017).
} 
in housing prices. Guren (2016) develops a model of housing price momentum, building on the incentive of individual sellers not to set a unilaterally high or low list price because the demand curve they face is concave in the relative price. In contrast to these models, informational frictions in our framework anchor on the interaction between the demand and supply sides of the housing market (rather than treating them as mutually independent), and feed back to both housing prices and real outcomes. This key feature is also distinct from the amplification of price volatility induced by dispersed information and short-sale constraints featured in Favara and Song (2014).

\section{The Model}

The model has two periods $t \in\{1,2\}$ and a single neighborhood. ${ }^{3}$ We interpret a neighborhood conceptually as a physical location in which households locate close to each other to benefit from their physical proximity, which could, in principle, be as broad as a Metropolitan Statistical Area (MSA) or a city. There are three types of agents in the economy: households looking to buy homes in the neighborhood, home builders, and capital producers. Suppose that this neighborhood is new and that all households purchase houses from home builders in a centralized market at $t=1$ after choosing whether to live in it. Households choose their labor supply and demand for capital, such as office space and warehouses, to complete production, and trade and consume consumption goods at $t=2$. Our intention is to capture the decision of a generation of home owners to move into a neighborhood. While static, our two period setting can represent a long period in which they live together and share amenities, as well as exchange their goods and services.

\subsection{Households}

We consider a pool of households, indexed by $i \in[0,1]$, each of which can choose either to live in or outside the neighborhood. Similar to Glaeser, Gyourko, and Saiz (2008), we consider only a single neighborhood and model this decision as a one-time option, with the reservation utility of living outside the neighborhood normalized for all households to zero. One can interpret the reservation utility as the expected value of paying a search cost to get a draw of productivity from another potential neighborhood. We can divide the unit interval

\footnotetext{
${ }^{3}$ For simplicity and tractability, our model features only a single neighborhood with a fixed outside option. In doing so, we abstract from the rich cross-sectional implications that arise in spatial equilibrium models such as Rosen (1979), Roback (1982), and Van Nieuwerburgh and Weill (2010).
} 
into the partition $\{\mathcal{N}, \mathcal{O}\}$, with $\mathcal{N} \cap \mathcal{O}=\varnothing$ and $\mathcal{N} \cup \mathcal{O}=[0,1]$. Let $H_{i}=1$ if household $i$ chooses to live in the neighborhood, i.e., $i \in \mathcal{N}$, and $H_{i}=0$ if it chooses to live elsewhere. If household $i$ at $t=1$ chooses to live in the neighborhood, it must purchase one house at price $P$. This reflects, in part, that housing is an indivisible asset and a discrete purchase, consistent with the insights of Piazzesi and Schneider (2009).

A key feature of housing markets is that households who locate near each other benefit from each other's goods and services, for instance, by patronizing each other's restaurants, shopping at each other's groceries, attending each other's schools, and seeking each other's medical and legal services. These goods and services from "non-tradable" industries rely on local demand and represent the social interactions underpinning the complementarity in housing choice that leads households of similar income, ideology, and/or socioeconomic status to live in the same neighborhood. Such complementarity also captures the agglomeration and spillover effects from households and firms locating near each other.

To incorporate this complementarity, we adopt a particular structure for their goods consumption and trading. Each household in the neighborhood produces a distinct good from the other households. Household $i$ has a Cobb-Douglas utility function over consumption of its own good $C_{i}(i)$ and its consumption of the goods produced by all other households in the neighborhood $\left\{C_{j}(i)\right\}_{j \in \mathcal{N} / i}$ :

$$
U\left(\left\{C_{j}(i)\right\}_{j \in \mathcal{N}} ; \mathcal{N}\right)=\left(\frac{C_{i}(i)}{1-\eta_{c}}\right)^{1-\eta_{c}}\left(\frac{\int_{\mathcal{N} / i} C_{j}(i) d j}{\eta_{c}}\right)^{\eta_{c}}
$$

The parameter $\eta_{c} \in(0,1)$ measures the weights of different consumption components in the utility function. A higher $\eta_{c}$ indicates a stronger complementarity between household $i^{\prime} s$ consumption of its own good and its consumption of the composite good produced by the other households in the neighborhood. ${ }^{4}$ This utility specification implies that each household cares about the strength of the neighborhood, i.e., the productivity of other households in the neighborhood. This assumption leads to strategic complementarity in households' housing demand, an important feature emphasized by the empirical literature, such as in Ioannides and Zabel (2003). ${ }^{5}$

\footnotetext{
${ }^{4}$ Alternatively, this complementarity could reflect that households and firms require each other's intermediate goods and services as inputs to their own production. Similar specifications of this utility function are employed, for instance, in Dixit and Stiglitz (1977) and Long and Plosser (1987) to give rise to input and output linkages in sectoral production. One can view $\left(\frac{1}{1-\eta_{c}} C_{i}(i)\right)^{1-\eta_{c}}\left(\frac{1}{\eta_{c}} \int_{\mathcal{N} / i} C_{j}(i) d j\right)^{\eta_{c}}$ as a final good produced by household $i$ given intermediate goods $\left\{C_{j}(i)\right\}_{i \in \mathcal{N}}$.

${ }^{5}$ While our model builds on complementarity in household consumption, other types of social interac-
} 
The production function of household $i$ is also Cobb-Douglas:

$$
e^{A_{i}} K_{i}^{\alpha} l_{i}^{1-\alpha},
$$

where $A_{i}$ is its productivity, $l_{i}$ is the household's labor choice, and $K_{i}$ is its choice of capital with a share of $\alpha \in(0,1)$ in the production function. We broadly interpret capital as both public and private investment in the neighborhood, which can include office, warehouses, and other equipment and infrastructure households can use for their productive activities. ${ }^{6}$ As we describe later, households buy capital from capital producers. When households are more productive in the neighborhood, the marginal productivity of capital is higher, and consequently capital producers are able to sell more capital at higher prices. Introducing capital allows us to discuss how learning affects the price and supply of not only residential housing, but also of local investment in the neighborhood.

Household $i$ 's productivity $A_{i}$ is comprised of a component $A$, common to all households in the neighborhood, and an idiosyncratic component $\varepsilon_{i}$ :

$$
A_{i}=A+\varepsilon_{i},
$$

where $A \sim \mathcal{N}\left(\bar{A}, \tau_{A}^{-1}\right)$ and $\varepsilon_{i} \sim \mathcal{N}\left(0, \tau_{\varepsilon}^{-1}\right)$ are both normally distributed and independent of each other. Furthermore, we assume that $\int \varepsilon_{i} d \Phi\left(\varepsilon_{i}\right)=0$ by the Strong Law of Large Numbers. The common productivity, $A$, represents the strength of the neighborhood, as a higher $A$ implies a more productive neighborhood. As $A$ determines the households' aggregate demand for housing, it also represents the demand-side fundamental.

As a result of realistic informational frictions, $A$ is not observable to households at $t=1$ when they need to make the decision of whether to live in the neighborhood. Instead, each household observes its own productivity $A_{i}$, after examining what it can do if it chooses to live in the neighborhood. Intuitively, $A_{i}$ combines the strength of the neighborhood $A$ and the household's own attribute $\varepsilon_{i}$. Thus, $A_{i}$ also serves as a noisy private signal about $A$ at $t=1$, as the household cannot fully separate its own attribute from the opportunity provided by the neighborhood. The parameter $\tau_{\varepsilon}$ governs both the household diversity in the neighborhood and the precision of this private signal. As $\tau_{\varepsilon} \rightarrow \infty$, the households' signals become infinitely

tions between households in a neighborhood may also lead to complementarity in their housing demand, as discussed in Durlauf (2004) and Glaeser, Sacerdote, and Scheinkman (2003).

${ }^{6}$ In the case that $K$ is a public good, its price can be interpreted as the tax a local government that faces a balanced budget can raise to offset the cost of construction. Our model then has implications for how housing markets impact the fiscal policy of local governments. 
precise and the informational frictions about $A$ vanish. Households care about the strength of the neighborhood because of complementarity in their demand for consumption. While a household may have a fairly good understanding of its own productivity when moving into a neighborhood, complementarity in consumption demand motivates it to pay attention to housing prices to learn about the average level of productivity $A$ for the neighborhood.

We start with each household's problem at $t=2$ and then work backward to describe its problem at $t=1$. At $t=2, A$ is revealed to all agents and we assume that each household experiences a disutility for supply labor $l_{i}^{1+\psi} /(1+\psi)$. A household in the neighborhood (i.e., $i \in \mathcal{N})$ maximizes its utility at $t=2$ by choosing labor $l_{i}$, capital $K_{i}$, and its consumption demand $\left\{C_{j}(i)\right\}_{j \in \mathcal{N}}$ :

$$
\begin{aligned}
U_{i}= & \max _{\left\{\left\{C_{j}(i)\right\}_{j \in \mathcal{N}}, l_{i}, K_{i}\right\}} U\left(\left\{C_{j}(i)\right\}_{j \in \mathcal{N}} ; \mathcal{N}\right)-\frac{l_{i}^{1+\psi}}{1+\psi} \\
& \text { such that } p_{i} C_{i}(i)+\int_{\mathcal{N} / i} p_{j} C_{j}(i) d j+R K_{i}=p_{i} e^{A_{i}} K_{i}^{\alpha} l_{i}^{1-\alpha},
\end{aligned}
$$

where $p_{i}$ is the price of the good it produces and $R$ is the unit price of capital. Households behave competitively and take the prices of their goods as given.

At $t=1$, each household needs to decide whether to live in the neighborhood. In addition to their private signals, all households and capital producers observe a noisy public signal $Q$ about the strength of the neighborhood $A$ :

$$
Q=A+\tau_{Q}^{-1 / 2} \varepsilon_{Q}
$$

where $\varepsilon_{Q} \sim \mathcal{N}(0,1)$ is independent of all other shocks. As $\tau_{Q}$ becomes arbitrarily large, $A$ becomes common knowledge to all agents. This public signal could, for instance, be news reports or published statistics on local economic conditions.

In addition to the utility flow $U_{i}$ at $t=2$ from goods consumption and labor disutility, we assume that households have quasi-linear expected utility at $t=1$ and, similar to Glaeser, Gyourko, and Saiz (2008), incur a linear utility penalty equal to the housing price $P$ if they choose to buy a house in the neighborhood. ${ }^{7}$ All housing units are homogenous and have the same price. Given that households have Cobb-Douglas preferences over their consumption, they are effectively risk-neutral at $t=1$, and their utility flow is their expected payoff, or the

\footnotetext{
${ }^{7}$ For simplicity, our model does not incorporate resale of housing after $t=2$. As a result, we do not include the housing price $P$ into the household's budget constraint at $t=2$. Instead, we treat the housing as a separate linear utility cost at $t=1$ following Glaeser, Gyourko, and Saiz (2008).
} 
value of their final consumption bundle less the cost of housing. ${ }^{8}$ Each household is subject to a participation constraint that its expected utility from moving into the neighborhood $E\left[U_{i} \mid \mathcal{I}_{i}\right]-P$ must (weakly) exceed its reservation utility, which we normalize to 0 :

$$
\max \left\{E\left[U_{i} \mid \mathcal{I}_{i}\right]-P, 0\right\}
$$

The moving decision is made at $t=1$ subject to each household's information set $\mathcal{I}_{i}=$ $\left\{A_{i}, P, Q\right\}$, which includes its private productivity signal $A_{i}$, the public signal $Q$, and the housing price $P .^{9}$

\subsection{Capital Producers}

In addition to households, there is a continuum of risk-neutral capital producers that develop capital at $t=1$, and sells this capital to households for their production at $t=2$. Similar to many macroeconomic models, such as Bernanke, Gertler, and Gilchrist (1999), we model capital producers as a separate sector in the neighborhood, although we match their population with households to simplify aggregation. This introduces a market-wide supply curve for capital, and consequently a market-wide price, at $t=2$, while avoiding introducing a speculative retrade motive into households' capital accumulation decisions.

The representative producer cares about the price of capital at $t=2, R$, which depends on capital's marginal productivity. This, in turn, depends on the strength of the neighborhood, and which households choose to live in the neighborhood. As a consequence, the housing price in the neighborhood serves as a useful signal to the producer when deciding how much capital to develop at $t=1$. We assume that each capital producer can develop $K$ units of capital by incurring a convex effort cost $\frac{1}{\lambda} K^{\lambda}$, where $\lambda>1$.

While households buy capital from capital producers at $t=2$, capital producers must forecast this demand when choosing how much capital $K$ to develop at $t=1$, in order to maximize its expected profit:

$$
\Pi_{c}=\sup _{K} E\left[R K-\frac{1}{\lambda} K^{\lambda} \mid \mathcal{I}^{c}\right]
$$

\footnotetext{
${ }^{8}$ While we focus on a static setting, introducing dynamics would reinforce our amplification mechanism stemming from learning. Since future housing prices are related to aggregate productivity growth in the neighborhood, households most optimistic about moving into the neighborhood because of trading opportunities today would also be the most optimistic in speculating about the value of selling their house to other households in the future.

${ }^{9} \mathrm{We}$ do not include the volume of housing transactions in the information set as a result of a realistic consideration that, in practice, people observe only delayed reports of total housing transactions at highly aggregated levels, such as national or metropolitan levels.
} 
where $\mathcal{I}^{c}=\{P, Q\}$ is the public information set, which includes the housing price $P$ and the public signal $Q$. It then follows that the optimal choice of capital sets the marginal cost, $K^{\lambda-1}$, equal to the expected price, $E\left[R \mid \mathcal{I}^{c}\right]$ :

$$
K=E\left[R \mid \mathcal{I}^{c}\right]^{\frac{1}{\lambda-1}}
$$

The realized housing price affects the expectation of capital producers about the neighborhood's strength $A$, which, in turn, impacts their choice of how much capital to develop. As a consequence, in addition to altering the moving decision of potential household entrants, informational frictions in the housing market also distort investment in the neighborhood.

Introducing capital plays a key role in amplifying the effects of informational frictions in the neighborhood. While, in principle, we could characterize learning in housing markets without introducing capital (as the special case when $\alpha=0$ ), the inability of the capital supply to adjust at $t=2$, when the strength of the neighborhood $A$ is publicly known, introduces an important, persistent distortion to each household's production decision. At $t=2$, while households can adjust their labor choice to mitigate the rational mistake that either too many or too few households entered the neighborhood ex post, the inability for capital to adjust nevertheless distorts the marginal product of labor for households as a result of the informational frictions. Such overhang from the excessive production of capital is important for understanding the recent U.S. housing boom and bust cycle, as areas such as Las Vegas and Phoenix saw overbuilding of commercial real estate in addition to housing. This capital misallocation also protracts the reversal after the bust: even if learning occurs quickly, the limited reversibility of housing and capital delays the subsequent correction. ${ }^{10}$

\subsection{Home Builders}

There is a population of home builders, indexed on a continuum $[0,1]$, in the neighborhood. Builder $i \in[0,1]$ builds a single house subject to a disutility from labor

$$
e^{-\frac{1}{1+k} \omega_{i}} S_{i}
$$

where $S_{i} \in\{0,1\}$ is the builder's decision to build and

$$
\omega_{i}=\xi+e_{i}
$$

\footnotetext{
${ }^{10}$ Such reversals are also, in fact, likely to be asymmetric depending on whether the local economy over or under-reacted to the true demand fundamental: it is likely easier to adjust upward the level of housing and capital than to adjust downward since housing and capital often entail costly reversibility.
} 
is the builder's productivity, which is correlated across builders in the neighborhood through $\xi$. We assume that $\xi=k \zeta$, where $k>0$ is a constant parameter, and $\zeta$ represents an unobserved, common shock to building costs in the neighborhood. From the perspective of households and builders, $\zeta \sim \mathcal{N}\left(\bar{\zeta}, \tau_{\zeta}^{-1}\right)$. Then, $\xi=k \zeta$ can be interpreted as a common supply shock with normal distribution $\xi \sim \mathcal{N}\left(\bar{\xi}, k^{2} \tau_{\zeta}^{-1}\right)$ with $\bar{\xi}=k \bar{\zeta}$. Furthermore, $e_{i} \sim$ $\mathcal{N}\left(0, \tau_{e}^{-1}\right)$ such that $\int e_{i} d \Phi\left(e_{i}\right)=0$ by the Strong Law of Large Numbers.

At $t=1$, each builder maximizes his profit

$$
\Pi_{s}\left(S_{i}\right)=\max _{S_{i}}\left(P-e^{-\frac{1}{1+k} \omega_{i}}\right) S_{i}
$$

Since builders are risk-neutral, each builder's optimal supply curve is

$$
S_{i}=\left\{\begin{array}{l}
1 \text { if } P \geq e^{-\frac{k \zeta+e_{i}}{1+k}} \\
0 \text { if } P<e^{-\frac{k \zeta+e_{i}}{1+k}} .
\end{array}\right.
$$

The parameter $k$ measures the supply elasticity of the neighborhood, which can arise, for instance, from structural limitations to building or zoning regulation. In the housing market equilibrium, the supply shock $\xi$ not only affects the supply side of the housing market but also demand, as it acts as informational noise in the price signal when households use the price to learn about the common productivity $A$. The elasticity parameter $k$ determines the amount of this informational noise in the price signal. ${ }^{11}$

\subsection{Noisy Rational Expectations Cutoff Equilibrium}

Our model features a noisy rational expectations cutoff equilibrium, which requires clearing of the real estate and capital markets that is consistent with the optimal behavior of households, home builders, and capital producers:

- Household optimization: each household chooses $H_{i}$ at $t=1$ to solve its maximization problem in (3), and then chooses $\left\{\left\{C_{j}(i)\right\}_{i \in \mathcal{N}}, l_{i}, K_{i}\right\}$ at $t=2$ to solve its maximization problem in (2).

\footnotetext{
${ }^{11}$ Although convenient for tractability, our specification of the housing supply curve is not essential for our key insight. We could instead have considered a setting with three neighborhoods: one with a perfectly inelastic housing supply, one with a perfectly elastic housing supply, and one in which housing supply is price-elastic and subject to noisy supply shocks. As supply is fixed in the perfectly inelastic area, housing price reflects only demand fundamental, and fully reveals the neighborhood strength $A$. In the perfectly elastic area, housing price always equals the marginal cost of building, and contains no information about $A$. It is in the intermediate elasticity area, where housing price is driven by both demand and supply-side factors, households and capital producers face the most severe filtering problem in inferring $A$ from housing price, a key feature captured by our more stylized model of housing supply.
} 
- Capital producer optimization: the representative producer chooses $K$ at $t=1$ to solve its maximization problem in (4).

- Builder optimization: each builder chooses $S_{i}$ at $t=1$ to solve his maximization problem in (5).

- At $t=1$, the housing price $P$ clears the housing market:

$$
\int_{-\infty}^{\infty} H_{i}\left(A_{i}, P, Q\right) d \Phi\left(\varepsilon_{i}\right)=\int_{-\infty}^{\infty} S_{i}\left(\omega_{i}, P, Q\right) d \Phi\left(e_{i}\right),
$$

where each household's housing demand $H_{i}\left(A_{i}, P, Q\right)$ depends on its productivity $A_{i}$, the housing price $P$, and the public signal $Q$, and each builder's housing supply $S_{i}\left(\omega_{i}, P, Q\right)$ depends on its productivity $\omega_{i}$, the housing price $P$, and the public signal $Q$. The demand from households and supply from builders are integrated over the idiosyncratic components of their productivity $\left\{\varepsilon_{i}\right\}_{i \in[0,1]}$ and $\left\{e_{i}\right\}_{i \in[0,1]}$, respectively.

- At $t=2$, the consumption good price clears the market for each household's good:

$$
C_{i}(i)+\int_{\mathcal{N} / i} C_{i}(j) d j=e^{A_{i}} K_{i}^{\alpha} l_{i}^{1-\alpha}, \quad \forall i \in \mathcal{N},
$$

and the capital price $R$ clears the market for capital:

$$
\int_{\mathcal{N}} K_{i} d i=K \int_{\mathcal{N}} d i
$$

where $\int_{\mathcal{N}} d i$ represents the population of households that live in the neighborhood.

\section{Equilibrium}

In this section, we analyze the housing market equilibrium. We first analyze each household's optimization problem given in (2), by conjecturing that only households with productivity higher than a cutoff $A^{*}$ enter the neighborhood. We then derive a unique equilibrium cutoff $A^{*}$ that satisfies the clearing condition of the housing market. Finally, we verify at the end of the section that the derived cutoff equilibrium is the unique rational expectations equilibrium, in which the choice of each household to live in the neighborhood is monotonic with respect to its own productivity $A_{i}$. 


\subsection{Choices of Households and Capital Producers}

We first analyze the choices of households living in the neighborhood at $t=2$, after its strength $A$ has been revealed to the public and capital producers and home builders have chosen their supply of capital and housing at $t=1$. The following proposition describes the household's optimal consumption, labor, and capital choices at $t=1$. All proofs are relegated to the Appendix.

Proposition 1 Let $\varphi=\frac{1+\psi}{(1-\alpha) \psi+(1+\alpha \psi) \eta_{c}}$, then at $t=2$, households $i$ 's optimal goods consumption is

$$
C_{i}(i)=\left(1-\eta_{c}\right)(1-\alpha) e^{A_{i}} K_{i}^{\alpha} l_{i}^{1-\alpha}, C_{j}(i)=\frac{1}{\Phi\left(\sqrt{\tau_{\varepsilon}}\left(A-A^{*}\right)\right)} \eta_{c}(1-\alpha) e^{A_{j}} K_{j}^{\alpha} l_{j}^{1-\alpha}
$$

and the price of its good is

$$
p_{i}=E\left[e^{\varphi\left(A_{j}-A_{i}\right)} \mid A, A_{j} \geq A^{*}\right]^{\eta_{c}} .
$$

Its optimal labor and capital choices are

$$
\begin{aligned}
\log l_{i}= & l_{A} A+l_{s} A_{i}+l_{R} \log R+\frac{1}{1-\alpha} \frac{\eta_{c}}{\psi} \log E\left[e^{\varphi\left(A_{j}-A\right)} \mid A, A_{j} \geq A^{*}\right]+l_{0}, \\
\log K_{i}= & (1+\psi) l_{A} A+(1+\psi) l_{s} A_{i}+\frac{\psi+\alpha}{\alpha} l_{R} \log R \\
& +(1+\psi) l_{\Phi} \log E\left[e^{\varphi\left(A_{j}-A\right)} \mid A, A_{j} \geq A^{*}\right]+h_{0},
\end{aligned}
$$

where $l_{A}, l_{s}>0>l_{R}$ and $\frac{d l_{A}}{d \eta_{c}}>0>\frac{d l_{A}}{d \eta_{c}}$, and all coefficients are given in the Appendix. Furthermore, the expected utility of household $i$ at $t=1$ is given by

$$
E\left[U\left(\left\{C_{j}(i)\right\}_{j \in \mathcal{N}} ; \mathcal{N}\right)-\frac{l_{i}^{1+\psi}}{1+\psi} \mid \mathcal{I}_{i}\right]=(1-\alpha) \frac{\psi}{1+\psi} E\left[p_{i} e^{A_{i}} K_{i}^{\alpha} l_{i}^{1-\alpha} \mid \mathcal{I}_{i}\right]
$$

Proposition 1 shows that each household spends a fraction $1-\eta_{c}$ of its wealth (excluding housing wealth) on consuming its own good $C_{i}(i)$ and a fraction $\eta_{c}$ on goods produced by its neighbors $\int_{\mathcal{N} / i} C_{j}(i) d j$. Households value each other's goods as a result of the complementarity in their utility functions, and the price of a household's good is inversely determined by the level of its output relative to that of the rest of the neighborhood. A household's good is thus more valuable when the rest of the neighborhood is more productive.

Proposition 1 also reveals that each household's optimal choices of labor and capital are both log-linear in the strength of the neighborhood, the household's own productivity, and 
the logarithm of the capital price. The final (nonconstant) term is the average idiosyncratic productivity of households above the cutoff $A^{*}$, reflecting that only the households that are most productive choose to live in the neighborhood. The optimal labor choice and demand for capital are both increasing in the strength of the neighborhood, because a stronger neighborhood represents improved trading opportunities with its neighbors, while they are both decreasing in the price of capital.

We now discuss each household's decision on whether to live in the neighborhood at $t=1$ when it still faces uncertainty about $A$. As a result of its Cobb-Douglas utility, the household is effectively risk-neutral over its aggregate consumption, and its optimal choice reflects the difference between its expected utility from living in the neighborhood and the cost $P$ of buying a house in the neighborhood. Then, household $i$ 's neighborhood decision is given by

$$
H_{i}=\left\{\begin{array}{l}
1 \text { if } \quad(1-\alpha) \frac{\psi}{1+\psi} E\left[p_{i} e^{A_{i}} K_{i}^{\alpha} l_{i}^{1-\alpha} \mid \mathcal{I}_{i}\right] \geq P \\
0 \text { if } \quad(1-\alpha) \frac{\psi}{1+\psi} E\left[p_{i} e^{A_{i}} K_{i}^{\alpha} l_{i}^{1-\alpha} \mid \mathcal{I}_{i}\right]<P
\end{array}\right.
$$

This decision rule supports our conjecture to search for a cutoff strategy for each household, in which only households with productivity above a critical level $A^{*}$ enter the neighborhood. This cutoff is eventually solved as a fixed point in the equilibrium.

Given each household's equilibrium cutoff $A^{*}$ at $t=1$ and optimal choices at $t=2$ from Proposition 1, we impose market-clearing in the market for capital to derive its price $R$ at $t=2$. Capital producers forecast this price to choose how much capital to develop at $t=1$. These observations are summarized in the following proposition.

Proposition 2 Given $K$ units of capital developed by capital producers at $t=1$, the price of capital at $t=2$ takes the log-linear form:

$$
\begin{aligned}
\log R= & \frac{1+\psi}{\psi+\alpha} A-\frac{\psi(1-\alpha)}{\psi+\alpha} \log K+\frac{1+\psi}{\psi+\alpha} \eta_{c} \log E\left[e^{\varphi\left(A_{j}-A\right)} \mid A, A_{j} \geq A^{*}\right] \\
& +\frac{\psi(1-\alpha)}{\psi+\alpha} \log E\left[e^{\left(1-\eta_{c}\right) \varphi\left(A_{j}-A\right)} \mid A, A_{j} \geq A^{*}\right]+\log \alpha+\frac{1-\alpha}{\psi+\alpha} \log (1-\alpha) .
\end{aligned}
$$

The optimal supply of capital by capital producers at $t=1$ is given by

$$
\begin{aligned}
& \log K=\frac{\log E\left[e^{\frac{1+\psi}{\psi+\alpha} A} E\left[e^{\varphi\left(A_{j}-A\right)} \mid A, A_{j} \geq A^{*}\right]^{\frac{1+\psi}{\psi+\alpha} \eta_{c}} E\left[e^{\left(1-\eta_{c}\right) \varphi\left(A_{j}-A\right)} \mid A, A_{j} \geq A^{*}\right]^{\frac{\psi(1-\alpha)}{\psi+\alpha}} \mid \mathcal{I}^{c}\right]}{\lambda-\alpha \frac{1+\psi}{\psi+\alpha}} \\
& +\log \alpha+\frac{1-\alpha}{\psi+\alpha} \log (1-\alpha),
\end{aligned}
$$

where $\lambda-\alpha \frac{1+\psi}{\psi+\alpha}>0$. 
Proposition 2 reveals that the capital price at $t=2$ is increasing in the strength of the neighborhood and in the average idiosyncratic productivity of the households that choose to live in the neighborhood, i.e., the last two (nonconstant) terms of (8). As one would expect, it is also decreasing in the supply of capital chosen at $t=1$. Importantly, equation (9) shows that the optimal supply of capital at $t=1$ reflects the expectations of capital builders not only over the strength of the neighborhood, but also the impact on the pool of households that select into the neighborhood. Intuitively, a higher productivity cutoff for households to join the neighborhood raises both the price at which households charge each other for their goods, $p_{i}$, and the average marginal product of capital compared to that of the full population.

\subsection{Perfect-Information Benchmark}

In this subsection, we characterize a positive benchmark. With perfect information, all households, home builders, and capital producers observe the strength of the neighborhood $A$ at $t=1$ when making their respective decisions. ${ }^{12}$ Households will sort into the neighborhood according to a cutoff equilibrium determined by the net benefit of living in the neighborhood, which trades off the opportunity of trading with other households in the neighborhood with the price of housing. Despite the inherent nonlinearity of our framework, the following proposition summarizes a tractable, unique rational expectations cutoff equilibrium that is characterized by the solution to a fixed-point problem over the endogenous cutoff of entry into the neighborhood, $A^{*}$.

Proposition 3 In the absence of informational frictions, there exists a unique rational expectations cutoff equilibrium, in which the following hold:

1. Given that other households follow a cutoff strategy, household $i$ also follows a cutoff strategy in its moving decision such that

$$
H_{i}=\left\{\begin{array}{l}
1 \text { if } A_{i} \geq A^{*}(A, \xi) \\
0 \quad \text { if } A_{i}<A^{*}(A, \xi)
\end{array},\right.
$$

where $A^{*}(A, \xi)$ solves equation (23) in the Appendix.

\footnotetext{
${ }^{12}$ This perfect-information setting may not be a normative benchmark. It is not obvious that the perfectinformation setting is the "first-best" outcome, since households may over or under-coordinate their actions, e.g. Angeletos and Pavan (2007), or overreact to public signals, e.g. Angeletos and Pavan (2004), Amador and Weill (2010), in the presence of strategic complementarity.
} 
2. The cutoff productivity $A^{*}(A, \xi)$ is monotonically decreasing in $\xi$, and is increasing in $A$ if $\eta_{c}<\eta_{c}^{*}$ and hump-shaped in $A$ if $\eta_{c}>\eta_{c}^{*}$, where $\eta_{c}^{*}$ is given in (24) in the Appendix.

3. The population entering the neighborhood is monotonically increasing in both $A$ and $\xi$.

4. The housing price takes the following log-linear form:

$$
\log P=\frac{1}{1+k}\left(\sqrt{\frac{\tau_{\varepsilon}}{\tau_{e}}}\left(A-A^{*}\right)-\xi\right) .
$$

5. The housing price $P$ and the utility of the household with the cutoff productivity $A^{*}$ are increasing and convex in A.

Proposition 3 characterizes the unique rational expectations cutoff equilibrium in the perfect-information benchmark, and confirms the optimality of a cutoff strategy for each household's moving decision when other households adopt a cutoff strategy. Households sort based on their individual productivity into the neighborhood, with the more productive, who expect more gains from living in the neighborhood, entering and participating in production at $t=2$. This determines the supply of labor at $t=2$, and, through this channel, the price of capital at $t=2$.

The optimal cutoff $A^{*}(A, \xi)$, determined by equation (23), represents the productivity of the marginal household who is indifferent to entering the neighborhood. The benefit to the marginal household, the expected utility gain from producing and trading with other households, should be equal to the cost, or the housing price. With Cobb-Douglas preferences, this benefit is equal to the expected value of the marginal household's output from production, which is increasing in the marginal household's productivity. The housing price, in contrast, is decreasing in the marginal household's productivity, since the price is increasing in the size of the population flowing into the neighborhood. The upward sloping benefit and downward sloping cost gives rise to a unique cutoff productivity, and consequently to a unique rational expectations cutoff equilibrium.

The proposition also provides comparative statics of the equilibrium cutoff $A^{*}(A, \xi)$ and the population that enters the neighborhood. This cutoff is decreasing in $\xi$, since a lower housing price incentivizes more households to enter the neighborhood for a given neighborhood strength $A$. As a result, a higher population enters the neighborhood as $\xi$ increases. 
The relation between the cutoff and neighborhood strength $A$, in contrast, reflects two offsetting forces. On the one hand, a higher $A$ implies a higher housing price and a higher price of capital, which raises the cutoff productivity since it is now more expensive to live in the neighborhood; on the other, complementarity lowers the cutoff because the gains from trade for high realizations of $A$ partially offset the increase in prices. As a result, the cutoff is either increasing in $A$ when complementarity is low and hump-shaped when complementarity is sufficiently high. Regardless of whether the cutoff increases or is hump-shaped in $A$, the population that enters the neighborhood increases with $A$ because a higher $A$ shifts right the distribution of households more than it moves the cutoff.

Given a cutoff productivity $A^{*}(A, \xi)$, the housing price $P$ positively loads on the strength of the neighborhood $A$, since a higher $A$ implies stronger demand for housing, and loads negatively on the supply shock $\xi$. As one would expect, the cutoff $A^{*}$ enters negatively into the price. The higher the cutoff, the fewer the households that enter the neighborhood, and the lower housing demand. Despite its log-linear representation, the housing price is actually a generalized linear function of $\sqrt{\frac{\tau_{\varepsilon}}{\tau_{e}}} A-\xi$, since $A^{*}$ is an implicit function of $A$ and $\log P$.

\subsection{Equilibrium with Informational Frictions}

Having characterized the perfect-information benchmark, we now turn to the equilibrium in the presence of informational frictions. With informational frictions, at $t=1$ households and capital producers must now forecast the strength of the neighborhood $A$, and the price of capital $R$ at $t=2$. Each household's type $A_{i}$ serves as a private signal about the strength of the neighborhood $A$. The publicly observed housing price serves as a public signal. As the equilibrium housing price is a nonlinear function of $A$, it poses a significant challenge to our derivation of the learning of households and producers. Interestingly, the equilibrium housing price maintains the same functional form as in (10) for the perfect-information benchmark. As a result, the information content of the publicly observed housing price can be summarized by a sufficient statistic $z(P)$ that is linear in $A$ and the supply shock $\xi$ :

$$
z(P)=A-\sqrt{\frac{\tau_{e}}{\tau_{\varepsilon}}} \xi .
$$

In our analysis, we shall first conjecture this linear sufficient statistic, and then verify that it indeed holds in the equilibrium. This conjectured linear statistic helps to ensure tractability of the equilibrium, despite that the equilibrium housing price is highly nonlinear. 
By solving for the learning of households and capital producers based on the conjectured sufficient statistic from the housing price, and by clearing the aggregate housing demand from the households with the supply from home builders, we derive the housing market equilibrium. The following proposition summarizes this equilibrium.

Proposition 4 There exists a unique noisy rational expectations cutoff equilibrium in the presence of informational frictions, in which the following hold:

1. The housing price takes a log-linear form:

$$
\log P=\frac{1}{1+k}\left[\sqrt{\frac{\tau_{\varepsilon}}{\tau_{e}}}\left(A-A^{*}\right)-\xi\right]=\frac{1}{1+k}\left[\sqrt{\frac{\tau_{\varepsilon}}{\tau_{e}}}\left(z-A^{*}\right)-\bar{\xi}\right] .
$$

2. The posterior of household $i$ after observing housing price $P$, the public signal $Q$, and its productivity $A_{i}$ is Gaussian with the conditional mean $\hat{A}_{i}$ and variance $\hat{\tau}_{A}$ given by

$$
\begin{aligned}
& \hat{A}_{i}=\hat{\tau}_{A}^{-1}\left(\tau_{A} \bar{A}+\tau_{Q} Q+\frac{\tau_{\varepsilon}}{\tau_{e}} \tau_{\xi} z+\tau_{\varepsilon} A_{i}\right), \\
& \hat{\tau}_{A}=\tau_{A}+\tau_{Q}+\frac{\tau_{\varepsilon}}{\tau_{e}} \tau_{\xi}+\tau_{\varepsilon},
\end{aligned}
$$

and the posterior of capital producers, after observing housing price $P$ and the public signal $Q$, is also Gaussian with the conditional mean $\hat{A}^{c}$ and variance $\hat{\tau}_{A}^{c}$ given by

$$
\begin{aligned}
& \hat{A}^{c}=\hat{\tau}_{A}^{c-1}\left(\tau_{A} \bar{A}+\tau_{Q} Q+\frac{\tau_{\varepsilon}}{\tau_{e}} \tau_{\xi} z\right), \\
& \hat{\tau}_{A}^{c}=\tau_{A}+\tau_{Q}+\frac{\tau_{\varepsilon}}{\tau_{e}} \tau_{\xi} .
\end{aligned}
$$

3. Given that other households follow a cutoff strategy, household $i$ also follows a cutoff strategy in its moving decision

$$
H_{i}=\left\{\begin{array}{ll}
1 & \text { if } A_{i} \geq A^{*}(z, Q) \\
0 & \text { if } A_{i}<A^{*}(z, Q)
\end{array},\right.
$$

where $A^{*}(z, Q)$ is the unique root to equation (26) in the Appendix.

4. The supply of capital takes the form:

$$
\log K=\frac{1}{\lambda-\alpha \frac{1+\psi}{\psi+\alpha}} \log F\left(\hat{A}^{c}-A^{*}, \hat{\tau}_{A}^{c}\right)+\frac{\frac{1+\psi}{\psi+\alpha}}{\lambda-\alpha \frac{1+\psi}{\psi+\alpha}} A^{*}+k_{0},
$$

where $F\left(\hat{A}^{c}-A^{*}, \hat{\tau}_{A}^{c}\right)$ is given in the Appendix, and $\log K$ is increasing in the conditional belief of capital producers $\hat{A}^{c}$. 
5. The cutoff productivity $A^{*}$ is decreasing, while the population entering the neighborhood and the housing price $P$ are increasing, in the noise in the public signal $\varepsilon_{Q}$. These properties also hold with respect to z under a sufficient, although not necessary, condition that ${ }^{13}$

$$
\frac{1+k}{1+\frac{\tau_{e}}{\tau_{\varepsilon} \tau_{\zeta}}\left(\tau_{A}+\tau_{Q}\right) k} \geq \frac{\lambda-\alpha \frac{1+\psi}{\psi+\alpha}}{\alpha \frac{1+\psi}{\psi+\alpha}} \frac{\psi+\alpha+(1-\alpha) \eta_{c}}{\alpha\left(1-\eta_{c}\right)(1+\psi)} \sqrt{\frac{\tau_{\varepsilon}}{\tau_{e}}} .
$$

6. The equilibrium converges to the perfect-information benchmark in Proposition 3 as $\tau_{Q} \nearrow \infty$.

Proposition 4 confirms that, in the presence of informational frictions, each household will optimally adopt a cutoff strategy when other households adopt a cutoff strategy. Informational frictions make the household's equilibrium cutoff $A^{*}(z, Q)$ a function of

$$
z(P)=(1+k) \sqrt{\frac{\tau_{e}}{\tau_{\varepsilon}}} \log P+A^{*},
$$

which is the summary statistic of the publicly observed housing price $P$, and the public signal $Q$, rather than $A$ and $\xi$ as in the perfect-information benchmark. This equilibrium cutoff, determined by equation (26), is the key channel for informational frictions to affect the housing price, as well as each capital producer's decision to develop capital. We analyze the economic consequences of informational frictions in the next section.

We conclude this section by establishing that the cutoff equilibria we have characterized, both with informational frictions and with perfect information, is the unique rational expectations equilibria in the economy. Regardless of the housing policies of other households in the neighborhood, each household will follow a cutoff strategy, which establishes the uniqueness of the cutoff equilibrium, as summarized in the following proposition.

Proposition 5 The unique rational expectations cutoff equilibrium is the unique rational expectations equilibrium in the economy.

\section{Model Implications}

This section analyzes how informational frictions amplify noise through learning to affect housing markets and local investment. We analyze these learning effects across neighborhoods that differ in two dimensions: 1) supply elasticity $k$, and 2) the degree of consumption

\footnotetext{
${ }^{13}$ One may notice that a higher degree of complementarity, $\eta_{c}$, tightens the sufficient condition, while much of our analysis suggests that it amplifies the role of informational frictions. This is because the condition is not necessary, and is derived by omitting terms for which $\eta_{c}$ is relevant for amplifying the learning effect.
} 
complementarity in household utility $\eta_{c}$. As documented in Glaeser (2013), Davidoff (2013), and Nathanson and Zwick (2018), housing price boom and bust were most pronounced in areas that were not particularly constrained by the supply of land. In our model, supply elasticity plays an important and nuanced role in the distortionary effects of learning. It is instructive to consider two polar cases. When supply is infinitely inelastic (i.e., $k \rightarrow 0$ ), housing prices are only determined by the strength of the neighborhood $A$, and are thus fully revealing. In this case, there is not any distortion from learning. When supply is infinitely elastic (i.e., $k \rightarrow \infty$ ), prices converge to $\log P=-\zeta$, which is driven only by the supply shock. ${ }^{14}$ In this case, prices contain no information about demand, and there is no learning from price. These two polar cases determine that the distortions caused by learning from housing prices are humped-shaped with respect to supply elasticity. We will further characterize this pattern in this section.

In our model, households' consumption complementarity reinforces the effects of informational frictions. Without complementarity, a stronger neighborhood (i.e., higher $A$ ) leads to higher prices of both housing and capital, and thus deter households from entering the neighborhood. With complementarity, however, a stronger neighborhood can be more attractive to households, because it means that other households in the neighborhood are more productive and thus provide a better opportunity for trade. In the presence of informational frictions, complementarity gives each household a stronger incentive to learn about $A$, and thus amplifies the potential distortionary effects from such learning.

While we have analytical expressions for most equilibrium outcomes, the key equilibrium cutoff $A^{*}$ needs to be solved numerically from the fixed-point condition in equation (26). We therefore analyze the equilibrium properties of $A^{*}$ and other variables through a series of numerical illustrations, by using the following benchmark parameters:

$$
\begin{aligned}
\tau_{A} & =0.5, \tau_{\varsigma}=2.0, \tau_{\varepsilon}=0.2, \tau_{Q}=1.0, \eta_{c}=0.8 \\
\psi & =2.5, k=0.5, \lambda=1.1, \bar{A}=0, \bar{\zeta}=0 .
\end{aligned}
$$

For the Frisch elasticity of labor supply, we choose $\psi=2.5$, which is within the typical range found in the literature. We set $\tau_{\zeta}$ to be four-fold larger than $\tau_{A}$ to ensure that with perfect information, the log housing price variance is monotonically declining in supply elasticity, consistent with conventional wisdom. We set $\lambda=1.1$ to have capital be in elastic supply, and to avoid having strong convexity in its production function. Finally, we choose the

\footnotetext{
${ }^{14}$ Note from equation (26) that $A^{*}$ remains finite a.s. as $k \rightarrow \infty$, allowing us to take the limit.
} 
neighborhood fundamental and the supply-side shock $A=\zeta=-0.5$, though the qualitative patterns we show hold more generically for a wide range of values, and we set the baseline noise in the public signal $Q$ to 0 .

In our analysis, we first examine the effects of learning when household's production does not require capital, $\alpha=0$, and there is no capital investment. In the latter part of our analysis, we turn on the role of capital by selecting $\alpha=0.33$, a standard value for capital share in the overall economy, to show that capital investment not only enriches our model's implications but also amplifies the learning effects in the housing market.

\subsection{Equilibrium Cutoff}

The equilibrium cutoff productivity $A^{*}(z, Q)$ is the key channel for informational frictions and learning to affect the housing market, as it determines the population flow into the neighborhood. We analyze this channel by showing how the two determinants $z$ and $Q$ affects the equilibrium cutoff.

We first examine a noise shock to the public signal $Q$, which can be interpreted as noise in public information, as in Morris and Shin (2002), or more broadly as housing market optimism, as in Kaplan, Mitman, and Violante (2017) and Gao, Sockin and Xiong (2019). In the perfect-information benchmark, the public signal $Q$ has no impact on either the equilibrium cutoff $A^{*}$ or the housing price because both the demand-side fundamental $A$ and the supply-side shock $\xi$ are publicly observable. In the presence of informational frictions, however, $Q$ affects the equilibrium as it shapes the beliefs of households and capital producers. The equilibrium housing price in (12) demonstrates that

$$
\frac{\partial \log P}{\partial Q}=-\frac{1}{1+k} \sqrt{\frac{\tau_{\varepsilon}}{\tau_{e}}} \frac{\partial A^{*}}{\partial Q} .
$$

By affecting the households' expectations of $A$, and consequently their cutoff productivity to enter the neighborhood, the noise in the public signal $Q$ affects the population that enters the neighborhood and the equilibrium housing price $\log P: \frac{\partial A^{*}}{\partial Q}<0$ and $\frac{\partial P}{\partial Q}>0$, as proved in Proposition 4. Furthermore, $Q$ also affects the price of capital, as well as each capital producer's optimal choice of how much capital to develop.

Figure 1 illustrates how the cutoff responds to a noise shock to the public signal $Q$. The first row depicts $\frac{\partial A^{*}}{\partial Q}$ across different values of supply elasticity $k$ in the left panel and degree of complementarity $\eta_{c}$ in the right panel. A noise shock to $Q$ has no impact 

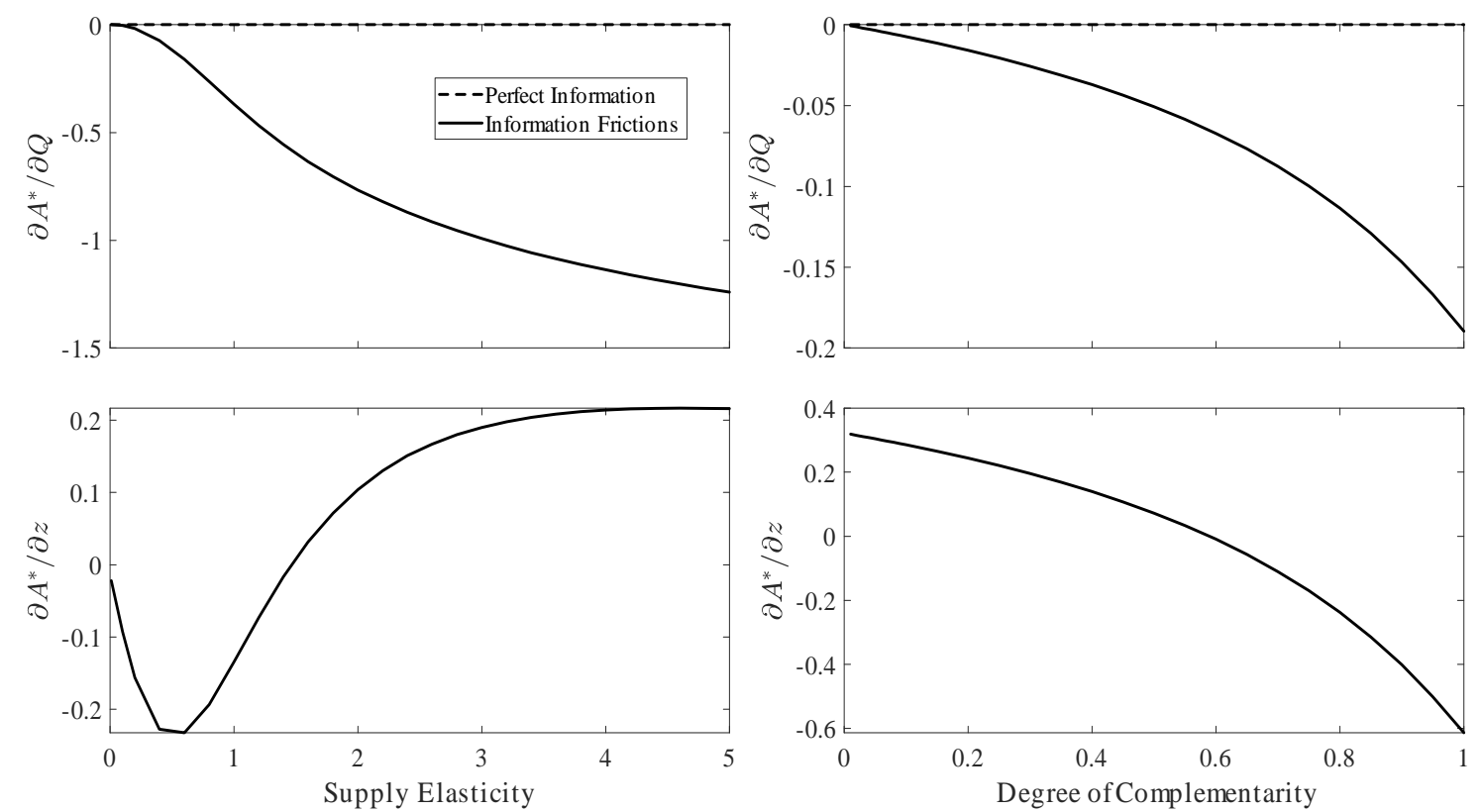

Figure 1: The response of the equilibrium cutoff productivity to a noise shock $Q$ (the first row) and a fundamental shock $z$ (the second row) across housing supply elasticity (left) and degree of complementarity (right). The dotted line in each panel is for the perfect-information benchmark, while the solid line is for the case with informational frictions.

on the equilibrium in the perfect-information benchmark. In the presence of informational frictions, however, the shock makes households more optimistic about $A$, and lowers the cutoff productivity $A^{*}$ for households to enter the neighborhood, as formally shown by Proposition 4, thus inducing a greater population flow into the neighborhood. Interestingly, this learning effect is stronger when supply elasticity is greater, or when the households' consumption complementarity is greater. The former results from the fact that a greater supply elasticity makes the housing price more dependent on supply-side factors, and therefore less informative about the neighborhood's strength $A$. Consequently, households place a greater weight on the public signal $Q$ in their learning about $A$, and this amplifies the effect of the noise shock to $Q$. The latter result is driven by the greater role that household learning plays as consumption complementarity increases, as a higher complementarity makes each household more concerned about the neighborhood strength.

In the presence of informational frictions, the demand-side fundamental $A$ and the supplyside shock $\xi$ are not directly observed by the public and, as a result, do not directly affect the housing price and other equilibrium variables. Instead, their equilibrium effects are bundled together in the housing price $P$ through the specific functional form of the sufficient statistic 
$z$. Consequently, a shock to $z$ may reflect a shock to either $A$ or $\xi$. The equilibrium housing price in (12) directly implies that the impact of a price shock is determined by its impact on the equilibrium cutoff $A^{*}$ :

$$
\frac{\partial \log P}{\partial z}=\frac{1}{1+k} \sqrt{\frac{\tau_{\varepsilon}}{\tau_{e}}}\left(1-\frac{\partial A^{*}}{\partial z}\right) .
$$

That is, depending on the sign of $\frac{\partial A^{*}}{\partial z}$, the equilibrium cutoff $A^{*}$ may amplify or dampen the impact of the $z$ shock on the housing price. Proposition 4 provides a sufficient (although not necessary) condition for $\frac{\partial A^{*}}{\partial z}<0$. In this case, there is an amplification effect. This amplification effect makes housing prices more volatile, as highlighted by Albagli, Hellwig, and Tsyvinski (2015) in their analysis of the cutoff equilibrium in an asset market. ${ }^{15}$

The second row of Figure 1 depicts $\frac{\partial A^{*}}{\partial z}$ across different values of supply elasticity $k$ in the left panel and degree of complementarity $\eta_{c}$ in the right panel. Interestingly, the left panel shows that $\frac{\partial A^{*}}{\partial z}$ has a U-shape with respect to supply elasticity. It is particularly negative when supply elasticity is in an intermediate value around 0.5 , and turns positive when supply elasticity rises roughly above 1.4. This U-shape originates from the aforementioned, non-monotonic learning effect of the housing price. Households use the housing price as a key source of information in their learning about the neighborhood strength $A$, and this learning effect is strongest when supply elasticity has an intermediate value, which makes the equilibrium cutoff particularly sensitive to the $z$ shock. The negative value of the effect implies that the cutoff productivity falls in response to the better neighborhood fundamental, resulting in more households entering the neighborhood despite the higher housing price. The right panel further illustrates that $\frac{\partial A^{*}}{\partial z}$ decreases monotonically with the degree of complementarity. Specifically, $\frac{\partial A^{*}}{\partial z}$ is positive when complementarity is low, and becomes negative as complementarity rises. This pattern confirms our earlier intuition that the learning effect from housing price strengthens with complementarity.

One could, in principle, directly test the effects of learning on population flows across different regions with properly designed measures of these non-fundamental shocks. Our analysis would suggest that non-fundamental shocks, such as the noisy demand shock, have a greater impact in inducing stronger population inflow to areas with greater degree of

\footnotetext{
${ }^{15}$ This interesting feature also differentiates our cutoff equilibrium from other type of nonlinear equilibrium with dispersed information, such as the log-linear equilibrium developed by Sockin and Xiong (2015) to analyze commodity markets. In their equilibrium, prices become less sensitive to their analogue of $z$ in the presence of informational frictions. This occurs because households, on aggregate, underreact to the fundamental shock in their private signals because of noise.
} 

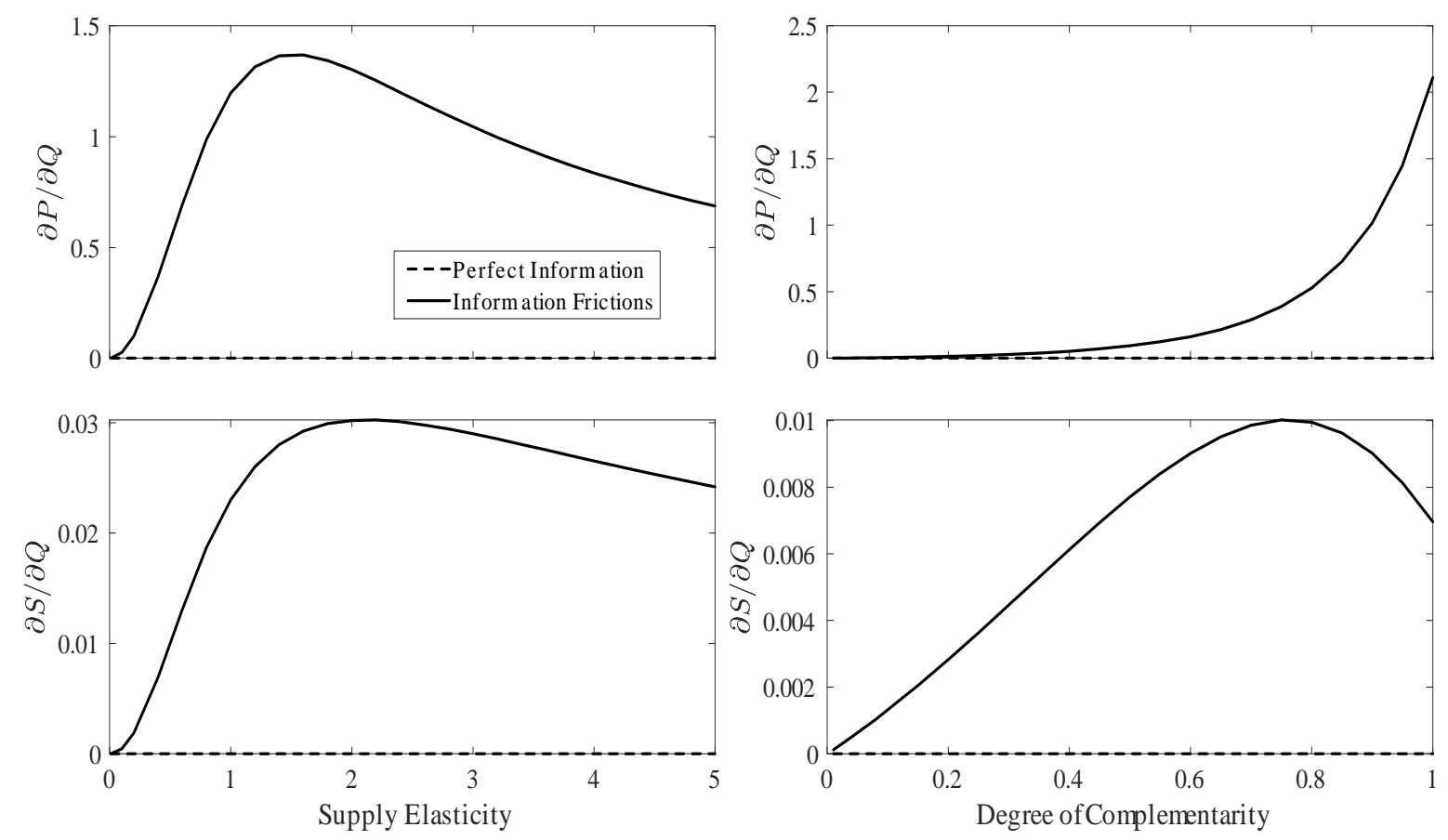

Figure 2: The responses of housing price $P$ (top row) and housing stock $S$ (bottom row) to a noise shock to the public signal $Q$ across supply elasticity (left) and degree of complementarity (right). The dotted line in each panel is for the perfect-information benchmark, while the solid line for the case with informational frictions.

complementarity and supply elasticity. Our model also implies that fundamental shocks, stemming either from the demand side or the supply side of the local housing market, have a greater impact on population flow to areas with greater degree of complementarity and intermediate supply elasticities. The differences in the cross-sectional patterns between the $Q$ and $z$ shocks can also help to distinguish between these two sources of optimism empirically.

\subsection{Housing Market}

We now analyze how informational frictions affect the housing market by examining the reactions of the housing market to two different shocks, a noise shock to $Q$ and a shock to housing supply $\zeta$. We interpret the deviations of the housing price in the presence of information frictions from the perfect-information benchmark as a measure of the amplification of noise because of learning.

Figure 2 illustrates the impacts of a noise shock to $Q$ on the housing price $P$ and housing stock $S=\int S_{i} d i$, by computing their partial derivatives with respect to $Q$ across different 
values of supply elasticity $k$ in the two left panels, and across different values of the degree of consumption complementarity $\eta_{c}$ in the two right panels. In the absence of informational frictions, this shock has no effect on the housing market. In the presence of informational frictions, the noise shock raises both the housing price and housing stock (Proposition 4) because it boosts agents' expectations about the neighborhood's strength $A$. Interestingly, the upper-left panel shows that this effect on the housing price is hump-shaped with respect to supply elasticity, and peaks at an intermediate value. This results from the non-monotonicity of the distortionary effect of learning. When housing supply is infinitely inelastic, the noise shock has a muted effect on households' expectations because the price is fully revealing. When housing supply is infinitely elastic, however, the housing price is fully determined by supply shock and is immune to households' learning about $A$. As a result, the price distortion caused by household learning is strongest when supply elasticity is in an intermediate range. The lower-left panel further shows that the impact of the noise shock on the housing stock has a similar hump-shaped pattern with supply elasticity.

The upper-right panel of Figure 2 shows that the effect of the noise shock on the housing price is increasing with respect to consumption complementarity. As complementarity rises, each household cares more about trading goods with other households, which makes households' expectations of the neighborhood strength a more influential determinant of the housing price. Consequently, the noise shock has a greater effect on the housing price. The pattern in housing stock (lower-right panel) is hump-shaped, reflecting that near perfect complementarity, almost all households choose to enter the neighborhood and the marginal effect of the increase in the equilibrium cutoff on neighborhood population diminishes.

Next, we analyze the effects of a shock to the housing price, which, as we discussed earlier, can be from either the demand side or the supply side. To avoid confusion in interpreting the results, we specifically examine a negative shock to the building $\operatorname{cost} \zeta$ (a negative supply shock). Figure 3 displays the responses of housing price $P$ and housing stock $S$ to this shock across different values of supply elasticity $k$ in the two left panels, and across different degrees of consumption complementarity $\eta_{c}$ in the two right panels. In the perfectinformation benchmark, the housing price increases with the negative supply shock, and the price increase rises with supply elasticity, as shown by the dashed line in the upper-left panel. In contrast, the lower-left panel shows that the housing stock falls with the negative supply shock since the higher housing price discourages more households from entering, and 

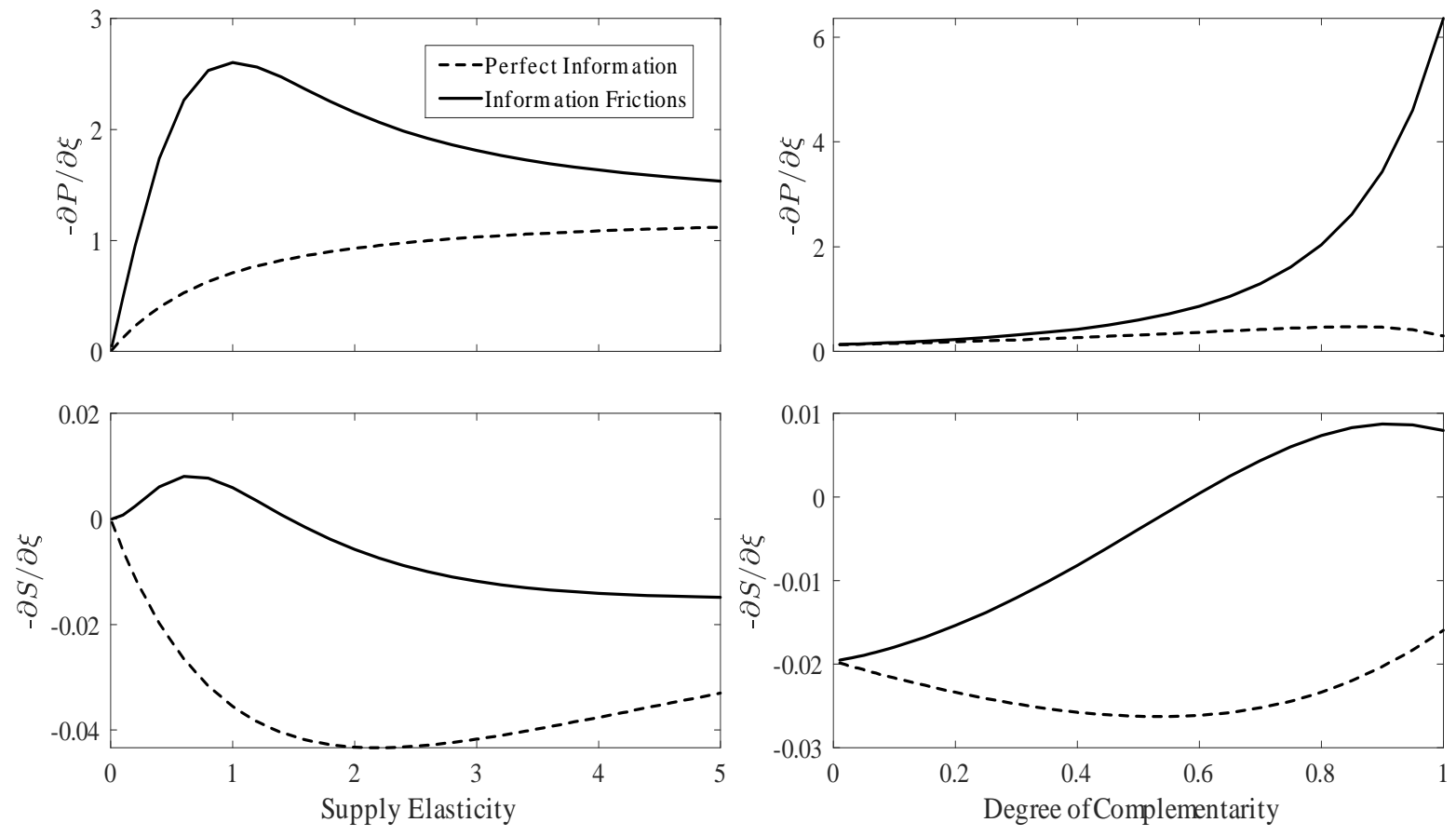

Figure 3: The responses of housing price $P$ (top row) and housing stock $S$ (bottom row) to a negative supply shock across supply elasticity (left) and degree of complementarity (right). The dotted line in each panel is for the perfect-information benchmark, while the solid line for the case with informational frictions.

the supply drop is greater when supply elasticity is larger.

In the presence of informational frictions, however, the negative supply shock is, in part, interpreted by households as a positive demand shock (i.e., stronger neighborhood $A$ ) when they observe a higher housing price. This learning effect, in turn, pushes up the housing price and the housing stock, relative to the perfect-information benchmark, as shown in the left panels of Figure 3. Across supply elasticity, these distortions are hump-shaped because the impact of learning from the housing price is most pronounced at intermediate supply elasticities, and, consequently, the response of the housing price and housing stock also peak at an intermediate range. As consumption complementarity increases, the learning effect from the negative supply shock is amplified, since households put more weight on the neighborhood's strength when determining whether to enter the neighborhood. This is shown in the upper-right panel of Figure 3. Similar to the $Q$ shock in Figure 2, the impact on the housing stock is hump-shaped, since most households are already entering the neighborhood as $\eta_{c}$ nears perfect complementarity.

Although our static model cannot deliver a boom-and-bust housing cycle across periods, 
one may intuitively interpret the deviation of the housing price induced by the positive $Q$ shock and the negative supply shock from its value in the perfect-information benchmark, as illustrated in Figures 2 and 3, as a price boom, which would eventually reverse. Then, we have testable cross-sectional implications for housing cycles - shocks, such as the noise shock and the supply shock, can lead to more pronounced housing cycles in areas with intermediate housing supply elasticities. Our model also implies that the magnitudes of housing price boom and bust are monotonically increasing with the degree of complementarity, while new housing supply has a hump-shaped relationship with the degree of complementarity. ${ }^{16}$

It is difficult in practice to directly measure volatility amplification and excessive volatility in housing markets. Nevertheless, these cross-sectional implications of our model are testable, and indirectly measure the excess volatility induced by learning. The non-monotonicity in these cross-sectional implications is particularly sharp, which motivates us to further explore the relationship of housing cycles with respect to supply elasticity and the degree of complementarity during the recent U.S. housing cycle in Section 4.

In addition to analyzing housing cycles, one could, in principle, also examine the crosssectional patterns we uncover for home buyer sentiment. The literature has suggested several empirical metrics of home buyer sentiment, such as the housing surveys in Case, Shiller, and Thompson (2012), Google search volume indices from Google Trends, and textual analysis of local media reports, as in Soo (2018). Whether informational frictions amplified noise originating from the demand or supply side of the housing market can, in principle, be disentangled by sorting these measures along the dimensions of supply elasticity and degree of complementarity, similar to the tests we propose using economic outcomes.

\subsection{Capital Investment}

Our analysis until now has abstracted from capital by setting $\alpha=0$. We now introduce for a role for capital by selecting $\alpha=0.33$, which allows us not only to extend the implications of our model to local real investment, such as in commercial real estate, but more importantly to show that capital investment can further amplify the impact of learning in housing markets.

\footnotetext{
${ }^{16}$ Since our model is not dynamic, however, we cannot speak to how informational frictions would impact the length of the boom or the bust that arises because of these noise or supply shocks. However, even if the informational frictions that give rise to a boom are short-lived, this does not necessarily imply that the impact of such frictions on the local economy are also short-lived. Since imperfect learning in our setting distorts the building of homes and the installation of capital, undoing this misallocation of resources can take years, such as with the overbuilding of homes in Las Vegas and office space in Phoenix during the recent U.S. housing cycle, as empirically examined by Gao, Sockin and Xiong (2019).
} 
We illustrate these effects in Figure 4 by building on our earlier analysis of the housing market's reaction to a negative supply shock. Specifically, the two left panels in Figure 4 correspond to the two left panels in Figure 3 with an additional, dotted line in each panel. This dotted line shows the reactions of the housing price and housing stock to the negative supply shock in the presence of capital investment (i.e. when $\alpha=0.33$ ). Interestingly, in the presence of capital investment, both the housing price and the housing stock react more strongly to the supply shock, while maintaining the same overall humped shapes with respect to supply elasticity. We also find a similar amplification of the reactions of the housing price and housing stock to a shock to $Q$, which we omit for brevity. Capital investment amplifies the housing market reactions to these shocks because capital producers make their investment decisions at $t=1$ when their expectations of the neighborhood strength are distorted by informational frictions. Their capital investment overhangs on the local economy at $t=2$, even though the neighborhood strength $A$ becomes observable to households and households flexibly adjust their labor supply. This capital overhang implies that households have access to cheap capital at $t=2$ when the market's expectations are overly optimistic at $t=1$. The anticipation of such access to capital motivates households to be even more aggressive in the housing market, which further amplifies the response of the housing price and housing stock to the shocks at $t=1$.

Figure 4 also shows how the price and stock of capital at $t=1$ react to the negative housing supply shock across different values of supply elasticity $k$ in the two right panels. We denote $R_{1}=E\left[R \mid \mathcal{I}^{c}\right]$, the expectation of capital producers at $t=1$ regarding $R$ the price of capital at $t=2$. This expectation determines the stock of capital $K_{S}$ they produce at $t=1$. In the perfect-information benchmark, the negative supply shock only impacts the housing price, and, through this channel, the cutoff productivity of the households that enter the neighborhood. This direct effect has only a modest impact on the market for capital. In the presence of informational frictions, however, its impact on the market for capital is substantially larger. This occurs because the negative supply shock is partially interpreted by capital producers as a positive shock to the strength of the neighborhood. Consequently, it distorts agents' expectations about $A$ upward, leading to overoptimism about the local economy. This results in both a higher capital price $R_{1}$ and a larger supply of capital $K_{S}$ at $t=1$. The magnitudes of these effects are all hump-shaped with respect to housing supply elasticity, as a result of the hump-shaped distortion to agents' expectations that arises from 

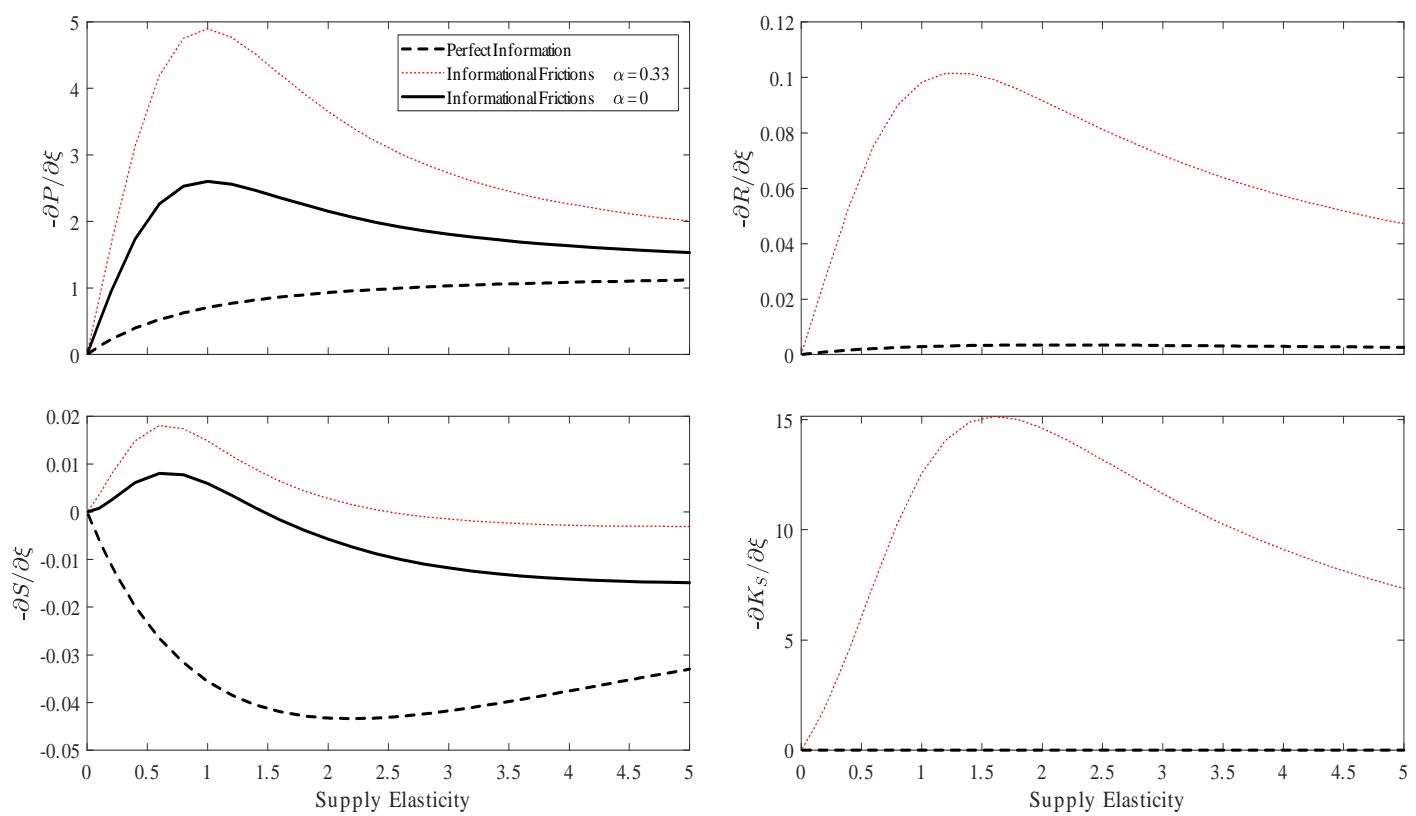

Figure 4: The responses of housing price $P$ (upper-left panel), housing stock $S$ (lower-left panel), capital price $R_{1}$ (upper-right panel), and capital stock $K$ (lower-right panel) to a negative housing supply shock $\xi$. In each panel, the dashed line is for the perfect-information benchmark, the solid line for the case with informational frictions and $\alpha=0$, while the dotted line for the case with informational frictions and $\alpha=0.33$.

their learning from the housing price.

Our analysis thus shows that shocks to the housing market can lead not only to a housing cycle, but also to a boom and bust in local investment. This concurrent boom and bust is consistent with Gyourko (2009) and Levitin and Wachter (2013), who highlight that the recent U.S. housing cycle was accompanied by a similar boom and bust in commercial real estate. It is difficult to simply attribute this commercial real estate boom to the subprime credit expansion, which was mainly targeted at households. In addition, while a run-up in the housing market can inflate commercial real estate prices if there is scarcity in developable land, as in Rosen (1979) and Roback (1982), such a boom would crowd out commercial real estate investment if it is driven by non-fundamental demand. In contrast, both the housing and commercial real estate markets experienced an expansion in construction along with the run-up in prices during the mid-2000s. Our model provides a coherent explanation for the synchronized cycles in both housing and commercial real estate markets. Furthermore, our analysis shows that these two cycles may amplify each other. 


\section{Empirical Evidence}

In this section, we provide several stylized facts to illustrate empirical relevance of our model from the recent U.S. housing cycle of the 2000s. The national U.S. housing market underwent a significant boom and bust cycle in the 2000s with the national home price index increasing over 60 percent from 2000 to 2006, and then falling back to its 2000 level by 2010. Many factors, such as the Clinton-era initiatives to broaden home ownership, the low interest rate environment of the late 1990s and early 2000s, the inflow of foreign capital, and the increase in securitization and sub-prime lending, contributed to the initial housing boom. While a well-known phenomenon at the time, the magnitude of the housing cycle experienced in the cross-section of U.S. regions reflected idiosyncratic uncertainty about their underlying fundamentals, which is the focus of our analysis. ${ }^{17}$ We designate the boom period of the recent U.S. housing cycle as 2001-2006, and the bust period as 2007-2010. ${ }^{18}$ In what follows, we examine how the magnitude of the housing price cycle, which serves as a proxy for the amplification of volatility illustrated by our model, and the intensity of new housing construction vary across MSAs with different values of supply elasticity and degree of complementarity. The objective of our empirical analysis is not to formally test our model, but rather to show that the key implications of our model are consistent with cross-sectional patterns during the recent U.S. housing cycle.

Our MSA-level house price data come from the Federal Housing Finance Agency (FHFA) House Price Index (HPI), which are constructed from repeat home sales. We employ the commonly used housing supply elasticity measure constructed by Saiz (2010). This elasticity measure focuses on geographic constraints by defining undevelopable land for construction as terrain with a slope of 15 degrees or more and areas lost to bodies of water including seas, lakes, and wetlands. This measure provides an exogenous measure of supply elasticity, with a higher value if an area is less geographically restricted. Saiz's measure is available for 269 Metropolitan Statistical Areas (MSAs).

To measure the supply-side activity in local U.S. housing markets, we use building permits from the U.S. Census Bureau, which conducts a survey in permit-issuing places all over

\footnotetext{
${ }^{17}$ The regional uncertainty introduced by this national phenomenon is absent from the local boom and bust episodes throughout the 1970s and 1980s. While there are other national housing cycles in history, such as in the roaring 20's, data limitations restrict our attention to the most recent U.S. housing cycle.

${ }^{18}$ See Gao, Sockin and Xiong (2019) for another study that uses a similar dating convention for the U.S. housing cycle in the 2000s.
} 

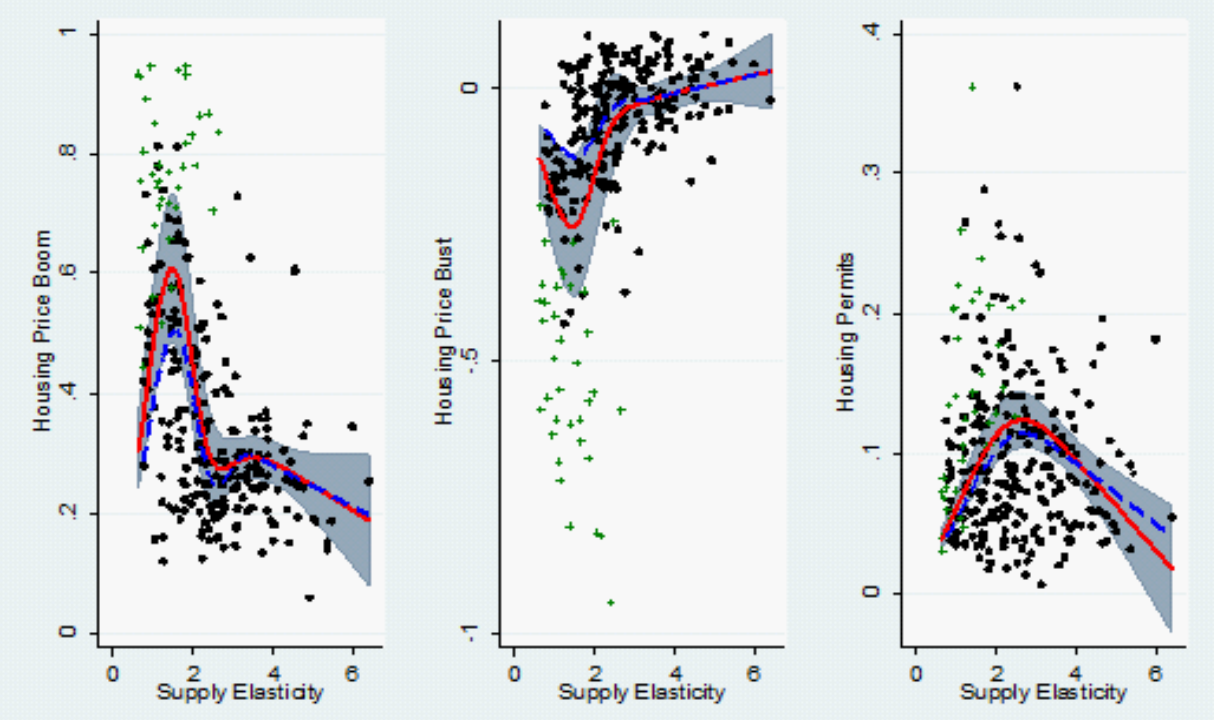

- Non-Sand + Sand

All Spline

No-Sand Spline

All $\mathrm{Cl}$

Figure 5: The U.S. housing cycle in 2000s across MSAs with different supply elasticities. The solid and cross dots represent MSAs outside and inside the sand states (Arizona, California, Florida and Neveda), respectively. The solid line is the spline line for all MSAs, while the dashed is for MSAs outside the sand states. $95 \%$ confidence intervals are displayed for the full sample. The standard errors are clustered at the state level.

the U.S. Compared with other construction-related measures, including housing starts and housing completions, building permits have detailed MSA-level information. In addition, building permits are issued right before housing starts and therefore can predict price trends in a timely manner. ${ }^{19}$ We measure new housing supply during the boom period by the building permits issued in 2001-2006 relative to the existing housing units in 2000 .

The first two panels of Figure 5 provide scatter plots of the housing price expansion and contraction experienced by each MSA during the housing boom and bust periods, respectively. To conveniently summarize the data, we include a spline line to fit each of the scatter plots (the solid line in the plots), together with $95 \%$ confidence interval (the shaded area around the spline line). These spline lines clearly indicate that the housing cycle was nonmonotonic with respect to supply elasticity - a hump-shaped pattern for the housing price

\footnotetext{
${ }^{19}$ Authorization to start is a largely irreversible process, with housing starts being only $2.5 \%$ lower than building permits at the aggregate level according to https://www.census.gov/construction/nrc/nrcdatarelationships.html, the website of the Census Bureau. Moreover, the delay between authorization and housing start is relatively short, on average less than one month, according to https://www.census.gov/construction/nrc/lengthoftime.html. These facts suggest that building permits are an appropriate measure of new housing supply.
} 
appreciation during the boom and a U-shaped pattern for the price drop during the bust. In particular, the cycle was most pronounced for MSAs with intermediate, rather than the lowest, supply elasticities.

One may be concerned that this non-monotonicity might be driven by the so-called "sand states" (Arizona, California, Florida, and Nevada). These four states experienced exceptional housing price booms and busts and, as several scholars including Davidoff (2013) and Nathanson and Zwick (2018) have noted, were characterized by peculiar speculative activities, such as land hoarding by real estate developers. In the scatter plots provided by Figure 5, we differentiate the MSAs in the sand states by "+" and provide a separate spline line (the dashed line in the plots) for observations excluding the sand-state MSAs. Indeed, the MSAs in the sand states experienced relatively more pronounced price appreciations during the boom and more severe price drops during the bust. Despite excluding these sandstate observations, the hump-shaped pattern for the price appreciation during the boom and the U-shaped pattern for the price drop during the bust remain significant, albeit with more attenuated magnitudes.

In addition to the housing price cycle, the third panel of Figure 5 provides a scatter plot of housing construction during the boom period, measured by new housing permits, with respect to supply elasticity. There is noticeably also a hump-shaped pattern with respect to supply elasticity, with MSAs in the intermediate elasticity range having the most new construction, instead of areas with the most elastic housing supply. This hump-shaped pattern is significant and robust to excluding the MSAs from the sand states. This surprising pattern in new construction has received little attention in the literature, and nicely supports our model implications.

Taken together, although common wisdom posits that supply elasticity attenuates housing cycles, we do not observe monotonic patterns across supply elasticity during the recent U.S. housing cycle in either the magnitude of the housing price boom and bust or in new construction. Instead, our analysis uncovers that MSAs with supply elasticities in an intermediate range experienced not only the most dramatic price cycles, but also the most new construction. While the number of MSAs in our sample is relatively small for a forceful test, these patterns nevertheless lend support to our key model implication that in the presence of informational frictions, volatility amplification induced by learning is most severe in areas with intermediate supply elasticity. 
Existing models of housing cycles have difficulty explaining these patterns. For example, Glaeser, Gyourko, and Saiz (2008) shows that, in the presence of housing supply constraints and extrapolative home buyer expectations, the overhang of housing supply developed during the boom may cause areas with intermediate supply elasticities to suffer most dramatic housing price drops during the subsequent bust. Their analysis, however, shows that housing price appreciation is decreasing while new construction is increasing across supply elasticity during the boom when the boom period is synchronized in the cross-section. Furthermore, while Nathanson and Zwick (2018) identify land speculation by real estate developers as an important mechanism driving the recent housing boom in intermediate elastic areas such as Las Vegas, their analysis does not provide a systematic theory for the full spectrum of housing cycles experienced across areas with different supply elasticities. In addition, the hoarding of land by optimistic developers in intermediate elastic areas, while exacerbating the housing price boom, has ambiguous implications about whether new construction is also the most pronounced in those MSAs.

We next provide additional evidence with respect to the cross-MSA relation between the housing cycle and a proxy for complementarity. Our model highlights complementarity as another important characteristic that amplifies the learning effects in housing markets. Specifically, Figures 2 and 3 show that, in the presence of informational frictions, there are monotonically increasing patterns in the effects of the noise shock and the negative supply shock on the magnitude of the housing price cycle with respect to complementarity, and hump-shaped patterns in the effects of these shocks on new housing supply during the boom. These patterns arise because complementarity, or the benefit from interacting with other households, exacerbates the feedback from learning in the presence of informational frictions.

We measure the degree of complementarity by non-tradable share of consumption in each MSA, as non-tradables are driven by local demand and thus reflect the complementarity in consumption of local residents. ${ }^{20}$ Specifically, we follow Mian and Sufi (2014) to identify non-tradable industries. Because non-tradable consumption data are not generally available across the U.S, we obtain the employment information across industries from County Business Pattern (CBP) data in the Census Bureau, aggregate the information to the MSA level, and calculate the share of employment in non-tradable industries as a proxy for consumption

\footnotetext{
${ }^{20}$ In unreported results, we have also used an alternative measure by the diversity of local industries, which give very similar patterns as those reported in Figure 6.
} 

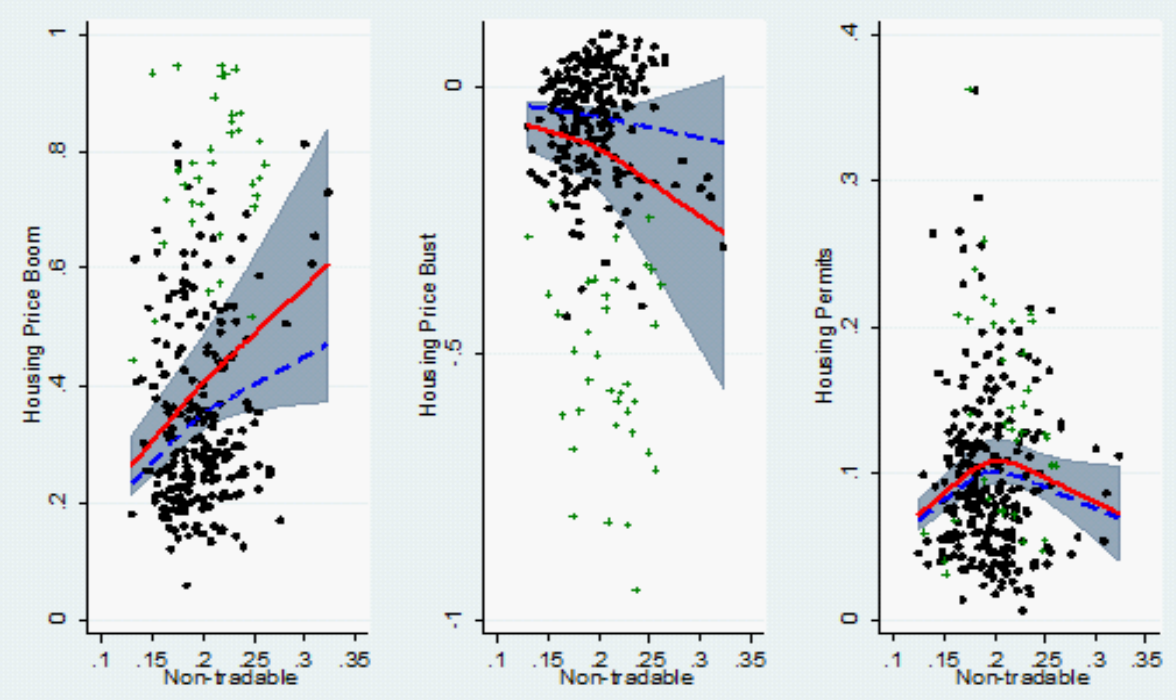

- Non-Sand + Sand

All Spline

No-Sand Spline

All Cl

Figure 6: The U.S. housing cycle in 2000s across MSAs with different degrees of complementarity. The solid and cross dots represent MSAs outside and inside the sand states (Arizona, California, Florida and Neveda), respectively. The solid line is the spline line for all MSAs, while the dashed is for MSAs outside the sand states. 95\% confidence intervals are displayed for the full sample. The standard errors are clustered at the state level.

complementarity. The higher is this ratio, local households rely more on local demand and stronger complementarity from each other.

The three panels in Figure 6 provide scatter plots of the housing price change during the boom period, the housing price change during the bust period, and new housing permits during the boom, respectively, against our measure of complementarity in different MSAs. The patterns match nicely with our model implications - the magnitudes of the price boom and bust appear to be monotonically increasing across complementarity, while new construction is hump-shaped. To the extent that these patterns cannot occur in the benchmark case with perfect information, Figure 6 confirms the empirical relevance of complementarity for the impact of learning on housing markets.

\section{Conclusion}

We introduce a model of information aggregation in housing markets, and examine its consequences for not only housing prices, but also for local economic outcomes such as new housing construction and real investment in capital. Our framework provides a novel amplification 
mechanism through learning in housing markets, and offers rich empirical predictions for the neighborhood's response across supply elasticity and the degree of complementarity to shocks originating from both demand and supply side factors in the presence of informational frictions. Such predictions can help rationalize the puzzling non-monotonic patterns that we uncover empirically across MSAs in the recent U.S. housing cycle.

\section{References}

Albagli, Elias, Christian Hellwig, and Aleh Tsyvinski (2015), A Theory of Asset Prices based on Heterogeneous Information, mimeo Bank of Chile, Toulouse School of Economics, and Yale University.

Albagli, Elias, Christian Hellwig, and Aleh Tsyvinski (2017), Imperfect Financial Markets and Shareholder Incentives in Partial and General Equilibrium, mimeo Bank of Chile, Toulouse School of Economics, and Yale University.

Albanesi, Stefania, Giacomo De Giorgi, and Jaromir Nosal (2017), Credit Growth and the Financial Crisis: A New Narrative, mimeo University of Pittsburgh, Geneva School of Economics and Management, and Boston College.

Amador, Manuel, and Pierre-Olivier Weill (2010), Learning from Prices: Public Communication and Welfare, Journal of Political Economy 118, 866-907.

Angeletos, George-Marios and Allesandro Pavan (2004), Transparency of Information and Coordination in Economies with Investment Complementarities, AEA Papers and Proceedings 94, 91-98.

Angeletos, George-Marios and Allesandro Pavan (2007), Efficient Use of Information and Social Value of Information, Econometrica 75, 1103-1142.

Bailey, Michael, Ruiqing Cao, Joannes Stroebel, and Theresa Kuchler (2017), The Economic Effects of Social Networks: Evidence from the Housing Market, Journal of Political Economy, forthcoming.

Bernanke, Ben S., Mark Gertler, and Simon Gilchrist (1999), The Financial Accelerator in a Quantitative Business Cycle Framework, Handbook of Macroeconomics 1, Edited by John B. Taylor and Michael Woodford, 1341-1393.

Bond, Philip, Alex Edmans, and Itay Goldstein (2012), The real effects of financial markets, Annuual Review of Financial Economics 4, 339-360.

Boyd, John H. (1990), Recursive Utility and the Ramsey Problem, Journal of Economic Theory, 50, 326-345.

Burnside, Craig, Martin Eichenbaum, and Sergio Rebelo (2016), Understanding Booms and Busts in Housing Markets, Journal of Political Economy 124(4): 1088-1147.

Case, Karl and Robert Shiller (2003), Is there a Bubble in the Housing Market?, Brookings Papers on Economic Activity 2003(2): 299-362. 
Case, Karl, Robert J. Shiller, and Anne Thompson (2012), What Have They Been Thinking? Homebuyer Behavior in Hot and Cold Markets, Brookings Papers on Economic Activity, 265-315.

Cheng, Ing-haw, Sahil Raina, and Wei Xiong (2014), Wall Street and the Housing Bubble, American Economic Review 104, 2797-2829.

Chinco, Alex, and Christopher Mayer (2015), Misinformed Speculators and Mispricing in the Housing Market, The Review of Financial Studies 29(2), 486-522.

Davidoff, Thomas (2013), Supply Elasticity and the Housing Cycle of the 2000s, Real Estate Economics 41(4), 793-813.

DeFusco, Anthony, Charles Nathanson, and Eric Zwick (2017), Speculative Dynamics of Prices and Volume. No. w23449. National Bureau of Economic Research.

Dixit, Avinash K. and Joseph E. Stiglitz (1977), Monopolistic Competition and Optimum Product Diversity, American Economic Review 67(3), 297-308.

Durlauf, Steven (2004), Neighborhood Effects, Handbook of Regional and Urban Economics $4,2173-2242$.

Favara, Giovanni, and Zheng Song (2014), House Price Dynamics with Dispersed Information, Journal of Economic Theory 149, 350-382.

Gao, Zhenyu, Michael Sockin, and Wei Xiong (2019), Economic Consequences of Housing Speculation, Review of Financial Studies, forthcoming.

Garmaise, Mark and Tobias Moskowitz (2004), Confronting Information Asymmetries: Evidence from Real Estate Markets, Review of Financial Studies 17, 405-437.

Glaeser, Edward (2013), A Nation of Gamblers: Real Estate Speculation and American History, American Economic Review Papers and Proceedings 103(3), 1-42.

Glaeser, Edward L., Joseph Gyourko, Eduardo Morales, and Charles G. Nathanson (2014), Housing Dynamics: An Urban Approach, Journal of Urban Economics 81, 45-56.

Glaeser, Edward, Joseph Gyourko, and Albert Saiz (2008), Housing Supply and Housing Bubbles, Journal of Urban Economics 64, 198-217.

Glaeser, Edward, Bruce Sacerdote, and José Scheinkman (2003), The Social Multiplier, Journal of the European Economics Association 1, 345-353.

Glaeser, Edward L., and Charles G. Nathanson (2015), Housing Bubbles, Handbook of Regional and Urban Economics. Vol. 5. Elsevier, 701-751.

Glaeser, Edward, and Charles Nathanson (2017), An Extrapolative Model of House Price Dynamics, Journal of Financial Economics, 126(1), 147-170

Goldstein, Itay, Emre Ozdenoren and Kathy Yuan (2013), Trading fFenzies and Their Impact on Real Investment, Journal of Financial Economics, 109(2), 566-582.

Griffin, John M., and Gonzalo Maturana (2015), Did Dubious Mortgage Origination Practices Distort House Prices?, Review of Financial Studies, forthcoming.

Grossman, Sanford and Joseph Stiglitz (1980), On the Impossibility of Informationally Efficient Markets, American Economic Review 70, 393-408. 
Guren, Adam (2016), House Price Momentum and Strategic Complementarity, Journal of Political Economy, forthcoming.

Gyourko, Joseph (2009), Understanding Commercial Real Estate: How Different from Housing Is It?, Journal of Portfolio Management 35, 23-37.

Hellwig, Martin (1980), On the Aggregation of Information in Competitive Markets, Journal of Economic Theory 22, 477-498.

Ioannides, Yannis and Jeffrey E. Zabel (2003), Neighborhood Effects and Housing Demand, Journal of Applied Econometrics 18, 563-584.

Kaplan, Greg, Kurt Mitman, and Gianluca Violante (2017), Consumption and house prices in the Great Recession: Model meets evidence, Manuscript, New York University.

Keys, Benjamin J., Tanmoy Mukherjee, Amit Seru, and Vikrant Vig (2009), Financial Regulation and Securitization: Evidence from Subprime Mortgage Loans, Journal of Monetary Economics 56 (5), 700-720.

Kurlat, Pablo and Johannes Stroebel (2014), Testing for Information Asymmetries in Real Estate Markets, mimeo, Stanford University and NYU.

Levitin, Adam J. and Susan M. Wachter (2013), The Commercial Real Estate Bubble, Harvard Business Law Review 3, 83-118.

Long, John B. and Jr. Plosser Charles I. (1987), Sectoral vs. Aggregate Shocks in the Business Cycle, American Economic Review 77, 333-336.

Mian, Atif, and Amir Sufi (2009). The Consequences of Mortgage Credit Expansion: Evidence from the US Mortgage Default Crisis, The Quarterly Journal of Economics, 124(4), 1449-1496.

Mian, Atif and Amir Sufi (2011), House Prices, Home Equity-Based Borrowing, and the US Household Leverage Crisis, American Economic Review 101, 2132-2156.

Mian, Atif and Amir Sufi (2014), What Explains the 2007-2009 Drop in Employment? Econometrica 82:2197-223.

Morris, Stephen and Hyun Song Shin (2002), The Social Value of Public Information, American Economic Review 92, 1521-1534.

Nathanson, Charles and Eric Zwick (2018), Arrested Development: Theory and Evidence of Supply-side Speculation in the Housing Market, Journal of Finance, forthcoming.

Piazzesi, Monika and Martin Schneider (2009), Momentum Traders in the Housing Market: Survey Evidence and a Search Model, American Economic Review Papers and Proceedings 99(2), 406-411.

Rosen, Sherwin (1979), Wage-Based Indexes of Urban Quality of Life, In Current Issues in Urban Economics, edited by Peter Mieszkowski and Mahlon Straszheim. Baltimore: Johns Hopkins University Press.

Roback, Jennifer (1982), Wages, Rents, and the Quality of Life, Journal of Political Economy $90,1257-1278$.

Saiz, Albert (2010), The Geographic Determinants of Housing Supply, Quarterly Journal of Economics 125(3), 1253-1296. 
Sockin, Michael and Wei Xiong (2015), Informational Frictions and Commodity Markets, Journal of Finance 70, 2063-2098

Soo, Cindy K. (2018), Quantifying Sentiment with News Media across Local Housing Markets, Review of Financial Studies 31(10), 3689-3719.

Van Nieuwerburgh, Stijn and Pierre-Olivier Weill (2010), Why Has House Price Dispersion Gone up?, Review of Economic Studies 77, 1567-1606.

\section{Appendix Proofs of Propositions}

\section{A.1 Proof of Proposition 1}

The first order conditions of household $i$ 's optimization problem in (2) respect to $C_{i}(i)$ and $C_{j}(i)$ at an interior point are

$$
\begin{aligned}
& C_{i}(i): \frac{1-\eta_{c}}{C_{i}(i)} U\left(\left\{C_{k}(i)\right\}_{k \in \mathcal{N}} ; \mathcal{N}\right)=\theta_{i} p_{i}, \\
& C_{j}(i): \frac{\eta_{c}}{\int_{\mathcal{N} / i} C_{j} d j} U\left(\left\{C_{k}(i)\right\}_{k \in \mathcal{N}} ; \mathcal{N}\right)=\theta_{i} p_{j},
\end{aligned}
$$

where $\theta_{i}$ is the Lagrange multiplier for the budget constraint. Rewriting (14) as

$$
\frac{\eta_{c} C_{j}}{\int_{\mathcal{N} / i} C_{j} d j} U\left(\left\{C_{k}(i)\right\}_{k \in \mathcal{N}} ; \mathcal{N}\right)=\theta_{i} p_{j} C_{j}
$$

and integrating over $\mathcal{N}$, we arrive at

$$
\eta_{c} U\left(\left\{C_{k}(i)\right\}_{k \in \mathcal{N}} ; \mathcal{N}\right)=\theta_{i} \int_{\mathcal{N} / i} p_{j} C_{j} d j
$$

Dividing equations (13) by this expression leads to $\frac{\eta_{c}}{1-\eta_{c}}=\frac{\int_{\mathcal{N} / i} p_{j} C_{j}(i) d j}{p_{i} C_{i}(i)}$, which in a symmetric equilibrium implies $p_{j} C_{j}(i)=\frac{1}{\Phi\left(\sqrt{\tau_{\varepsilon}}\left(A-A^{*}\right)\right)} \frac{\eta_{c}}{1-\eta_{c}} p_{i} C_{i}(i)$. By substituting this equation back to the household's budget constraint in (2), we obtain

$$
C_{i}(i)=\left(1-\eta_{c}\right)(1-\alpha) e^{A_{i}} K_{i}^{\alpha} l_{i}^{1-\alpha} .
$$

The market-clearing for the household's good requires that

$$
C_{i}(i)+\int_{\mathcal{N} / i} C_{i}(j) d j=(1-\alpha) e^{A_{i}} K_{i}^{\alpha} l_{i}^{1-\alpha}
$$


which implies that $C_{i}(j)=\frac{1}{\Phi\left(\sqrt{\tau_{\varepsilon}}\left(A-A^{*}\right)\right)} \eta_{c}(1-\alpha) e^{A_{i}} K_{i}^{\alpha} l_{i}^{1-\alpha}$.

The first order condition in equation (13) also gives the price of the good produced by household $i$. Since the household's budget constraint in (2) is entirely in nominal terms, the price system is only identified up to $\theta_{i}$, the Lagrange multiplier. We therefore normalize $\theta_{i}$ to 1 . It follows that

$$
p_{i}=\frac{1-\eta_{c}}{C_{i}(i)} U\left(\left\{C_{j}(i)\right\}_{j \in \mathcal{N}} ; \mathcal{N}\right)=\left(e^{A_{i}} K_{i}^{\alpha} l_{i}^{1-\alpha}\right)^{-\eta_{c}}\left(\frac{\int_{\mathcal{N} / i} e^{A_{j}} K_{j}^{\alpha} l_{j}^{1-\alpha} d j}{\Phi\left(\sqrt{\tau_{\varepsilon}}\left(A-A^{*}\right)\right)}\right)^{\eta_{c}}
$$

The first-order conditions for household $i$ 's choice of $l_{i}$ at an interior point is

$$
l_{i}^{\psi}=(1-\alpha) \theta_{i} p_{i} e^{A_{i}}\left(\frac{K_{i}}{l_{i}}\right)^{\alpha}
$$

from equation (13). Substituting $\theta_{i}=1$ and $p_{i}$ with equation (15), it follows that

$\log l_{i}=\frac{1}{\psi+\alpha+(1-\alpha) \eta_{c}} \log (1-\alpha)+\frac{1}{\psi+\alpha+(1-\alpha) \eta_{c}} \log \left(\left(e^{A_{i}} K_{i}^{\alpha}\right)^{\left(1-\eta_{c}\right)}\left(\frac{\int_{\mathcal{N} / i} e^{A_{j}} K_{j}^{\alpha} l_{j}^{1-\alpha} d j}{\Phi\left(\sqrt{\tau_{\varepsilon}}\left(A-A^{*}\right)\right)}\right)^{\eta_{c}}\right)$

The optimal labor choice of household $i$, consequently, represents a fixed point problem over the optimal labor strategies of other households in the neighborhood.

Noting that $K_{i}=\left(\frac{\alpha p_{i} e^{A_{i}} l_{i}^{1-\alpha}}{R}\right)^{\frac{1}{1-\alpha}}$ from the first-order condition for $K_{i}$, we can substitute in the price function $p_{i}$ to arrive at

$$
\begin{aligned}
\log K_{i}= & \frac{1}{1-\left(1-\eta_{c}\right) \alpha} \log \left(\left(e^{A_{i}} l_{i}^{1-\alpha}\right)^{1-\eta_{c}}\left(\frac{1}{\Phi\left(\sqrt{\tau_{\varepsilon}}\left(A-A^{*}\right)\right)} \int_{\mathcal{N} / i} e^{A_{j}} K_{j}^{\alpha} l_{j}^{1-\alpha} d j\right)^{\eta_{c}}\right) \\
& -\frac{1}{1-\left(1-\eta_{c}\right) \alpha} \log R+\frac{1}{1-\left(1-\eta_{c}\right) \alpha} \log \alpha
\end{aligned}
$$

which is a functional fixed-point problem for the optimal choice of capital. With some manipulation, by adding a multiple $\frac{1-\left(1-\eta_{c}\right) \alpha}{\psi+\alpha+(1-\alpha) \eta_{c}}$ of equation (18) to equation (17), we have

$$
\log K_{i}=(1+\psi) \log l_{i}-\log \alpha(1-\alpha)-\log R
$$

and substituting this back into equation (17), we arrive at the functional fixed-point equation

$$
\begin{aligned}
A_{i}+(1+\alpha \psi) \log l_{i}= & \frac{1+\psi}{(1-\alpha) \psi+(1+\alpha \psi) \eta_{c}} A_{i}-\frac{(1+\alpha \psi) \alpha}{(1-\alpha) \psi+(1+\alpha \psi) \eta_{c}}(\log \alpha(1-\alpha)+\log R) \\
& +\frac{1+\alpha \psi}{(1-\alpha) \psi+(1+\alpha \psi) \eta_{c}}\left(\log (1-\alpha)-\eta_{c} \log \Phi\left(\sqrt{\tau_{\varepsilon}}\left(A-A^{*}\right)\right)\right) \\
& +\frac{(1+\alpha \psi) \eta_{c}}{(1-\alpha) \psi+(1+\alpha \psi) \eta_{c}} \log \left(\int_{\mathcal{N} / i} e^{A_{j}} l_{j}^{1+\alpha \psi} d j\right) .
\end{aligned}
$$


Given that household $i$ 's optimal labor supply $l_{i}$ satisfies the functional fixed-point equation (19), let us conjecture for $i$ for which $A_{i} \geq A^{*}$, so that $i \in \mathcal{N}$ is in the neighborhood, that

$\log l_{i}=l_{0}+l_{A} A+l_{s} A_{i}+l_{R} \log R+l_{\Phi} \log \frac{e^{\frac{1}{2}\left(\frac{1+\psi}{(1-\alpha) \psi+(1+\alpha \psi) \eta_{c}}\right)^{2} \tau_{\varepsilon}^{-1}} \Phi\left(\left(1+\left(\alpha h_{s}+(1-\alpha) l_{s}\right)\right) \tau_{\varepsilon}^{-1 / 2}+\frac{A-A^{*}}{\tau_{\varepsilon}^{-1 / 2}}\right)}{\Phi\left(\sqrt{\tau_{\varepsilon}}\left(A-A^{*}\right)\right)}$,

where $R$ is the rental rate of capital. Substituting these conjectures into the fixed-point recursion for labor, equation (17), we arrive, by the method of undetermined coefficients, at the coefficient restrictions:

$$
\begin{aligned}
l_{0} & =\frac{1}{2} \frac{1}{1-\alpha} \frac{\eta_{c}}{\psi}\left(\frac{1+\psi}{(1-\alpha) \psi+(1+\alpha \psi) \eta_{c}}\right)^{2} \tau_{\varepsilon}^{-1}+\frac{\alpha}{1-\alpha} \frac{1}{\psi} \log \alpha+\frac{1}{\psi} \log (1-\alpha), \\
l_{A} & =\frac{1}{1-\alpha} \frac{1+\psi}{(1-\alpha) \psi+(1+\alpha \psi) \eta_{c}} \frac{\eta_{c}}{\psi}, \\
l_{s} & =\frac{1-\eta_{c}}{(1-\alpha) \psi+(1+\alpha \psi) \eta_{c}}, \\
l_{R} & =-\frac{\alpha}{1-\alpha} \frac{1}{\psi} \\
l_{\Phi} & =\frac{1}{1-\alpha} \frac{\eta_{c}}{\psi}
\end{aligned}
$$

which confirms the conjecture for $A_{i} \geq A^{*}$. It is straightforward to verify from these expressions that $\frac{d l_{A}}{d \eta_{c}}>0>\frac{d l_{A}}{d \eta_{c}}$. In addition, it follows that

$$
\begin{aligned}
\log K_{i}= & (1+\psi) l_{s} A_{i}+(1+\psi) l_{A} A+\frac{\psi+\alpha}{\alpha} l_{R} \log R \\
& +(1+\psi) l_{\Phi} \log e^{\frac{1}{2}\left(\frac{1+\psi}{(1-\alpha) \psi+(1+\alpha \psi) \eta_{c}}\right)^{2} \tau_{\varepsilon}^{-1}} \frac{\Phi\left(\frac{1+\psi}{(1-\alpha) \psi+(1+\alpha \psi) \eta_{c}} \tau_{\varepsilon}^{-1 / 2}+\frac{A-A^{*}}{\tau_{\varepsilon}^{-1 / 2}}\right)}{\Phi\left(\sqrt{\tau_{\varepsilon}}\left(A-A^{*}\right)\right)}+h_{0},
\end{aligned}
$$

Substituting this functional form for the labor supply and capital demand of household $i$ into equation (15), the price of household $i^{\prime} s$ good then reduces to

$$
p_{i}=e^{\frac{1+\psi}{(1-\alpha) \psi+(1+\alpha \psi) \eta_{c}}} \eta_{c}\left(A-A_{i}\right)+\frac{1}{2} \eta_{c}\left(\frac{1+\psi}{(1-\alpha) \psi+(1+\alpha \psi) \eta_{c}}\right)^{2} \tau_{\varepsilon}^{-1}\left(\frac{\Phi\left(\frac{1+\psi}{(1-\alpha) \psi+(1+\alpha \psi) \eta_{c}} \tau_{\varepsilon}^{-1 / 2}+\frac{A-A^{*}}{\tau_{\varepsilon}^{-1 / 2}}\right)}{\Phi\left(\sqrt{\tau_{\varepsilon}}\left(A-A^{*}\right)\right)}\right)^{\eta_{c}} .
$$

To arrive at the expressions in the statement of the proposition, we define $\varphi=\frac{1+\psi}{(1-\alpha) \psi+(1+\alpha \psi) \eta_{c}}$ and recognize that

$$
e^{\frac{1}{2} \varphi^{2} \tau_{\varepsilon}^{-1}} \frac{\Phi\left(\varphi \tau_{\varepsilon}^{-1 / 2}+\frac{A-A^{*}}{\tau_{\varepsilon}^{-1 / 2}}\right)}{\Phi\left(\sqrt{\tau_{\varepsilon}}\left(A-A^{*}\right)\right)}=E\left[e^{\varphi\left(A_{j}-A\right)} \mid A, A_{j} \geq A^{*}\right] .
$$

Furthermore, given equation (1), it follows since $C_{i}(i)=\left(1-\eta_{c}\right)(1-\alpha) e^{A_{i}} K_{i}^{\alpha} l_{i}^{1-\alpha}, C_{j}(i)=$ $\frac{1}{\Phi\left(\sqrt{\tau_{\varepsilon}}\left(A-A^{*}\right)\right)} \eta_{c}(1-\alpha) e^{A_{j}} K_{j}^{\alpha} l_{j}^{1-\alpha}$, and the optimal choice of $l_{i}$ that

$$
E\left[U\left(\left\{C_{j}(i)\right\}_{j \in \mathcal{N}} ; \mathcal{N}\right)-\frac{l_{i}^{1+\psi}}{1+\psi} \mid \mathcal{I}_{i}\right]=(1-\alpha) \frac{\psi}{1+\psi} E\left[p_{i} e^{A_{i}} K_{i}^{\alpha} l_{i}^{1-\alpha} \mid \mathcal{I}_{i}\right]
$$


from substituting with the household's budget constraint at $t=2$.

\section{A.2 Proof of Proposition 2}

Substituting the optimal demand for capital $K_{i}$ into the market-clearing condition for the capital in (7) reveals that the price $R$ is given by

$$
\begin{aligned}
\log R= & \frac{1+\psi}{\psi+\alpha} A-(1-\alpha) \frac{\psi}{\psi+\alpha} \log K+\log \alpha+\frac{1-\alpha}{\psi+\alpha} \log (1-\alpha) \\
& +\frac{1+\psi}{\psi+\alpha} \eta_{c} \log \frac{e^{\frac{1}{2}\left(\frac{1+\alpha) \psi+(1+\alpha \psi) \eta_{c}}{(1-\alpha)_{\varepsilon}^{-1}} \Phi\left(\frac{1+\psi}{(1-\alpha) \psi+(1+\alpha \psi) \eta_{c}} \tau_{\varepsilon}^{-1 / 2}+\frac{A-A^{*}}{\tau_{\varepsilon}^{-1 / 2}}\right)\right.}}{\Phi\left(\sqrt{\tau_{\varepsilon}}\left(A-A^{*}\right)\right)} \\
& +(1-\alpha) \frac{\psi}{\psi+\alpha} \log \frac{e^{\frac{1}{2}\left(1-\eta_{c}\right)^{2}\left(\frac{1+\psi}{(1-\alpha) \psi+(1+\alpha \psi) \eta_{c}}\right)^{2} \tau_{\varepsilon}^{-1}} \Phi\left(\frac{(1+\psi)\left(1-\eta_{c}\right)}{(1-\alpha) \psi+(1+\alpha \psi) \eta_{c}} \tau_{\varepsilon}^{-1 / 2}+\frac{A-A^{*}}{\tau_{\varepsilon}^{-1 / 2}}\right)}{\Phi\left(\sqrt{\tau_{\varepsilon}}\left(A-A^{*}\right)\right)}
\end{aligned}
$$

where $K$ is the total amount of capital developed by capital producers at $t=1$

Since market-clearing in the market for capital imposes that $K \int_{i \in \mathcal{N}} d i=\int_{i \in \mathcal{N}} K_{i} d i$, it follows from equation (4) that the optimal choice of how much capital that capital producers create is given by

$$
\begin{aligned}
\log K=\frac{1}{\lambda-\alpha \frac{1+\psi}{\psi+\alpha}} \log E & {\left[e^{\frac{1+\psi}{\psi+\alpha} A\left(\frac{\Phi\left(\frac{1+\psi}{(1-\alpha) \psi+(1+\alpha \psi) \eta_{c}} \tau_{\varepsilon}^{-1 / 2}+\frac{A-A^{*}}{\tau_{\varepsilon}^{-1 / 2}}\right)}{\Phi\left(\sqrt{\tau_{\varepsilon}}\left(A-A^{*}\right)\right)}\right)^{\frac{1+\psi}{\psi+\alpha} \eta_{c}}}\right.} \\
& \left.\left.\cdot\left(\frac{\Phi\left(\frac{(1+\psi)\left(1-\eta_{c}\right)}{(1-\alpha) \psi+(1+\alpha \psi) \eta_{c}} \tau_{\varepsilon}^{-1 / 2}+\frac{A-A^{*}}{\tau_{\varepsilon}^{-1 / 2}}\right)}{\Phi\left(\sqrt{\tau_{\varepsilon}}\left(A-A^{*}\right)\right)}\right)^{\frac{\psi(1-\alpha)}{\psi+\alpha}}\right] \mathcal{I}^{c}\right]+k_{0},
\end{aligned}
$$

with constant $k_{0}$ is given by

$$
k_{0}=\frac{\log \alpha+\frac{1-\alpha}{\psi+\alpha} \log (1-\alpha)+\frac{1}{2}\left(\frac{1+\psi}{\psi+\alpha} \eta_{c}+(1-\alpha) \frac{\psi}{\psi+\alpha}\left(1-\eta_{c}\right)^{2}\right)\left(\frac{1+\psi}{(1-\alpha) \psi+(1+\alpha \psi) \eta_{c}}\right)^{2} \tau_{\varepsilon}^{-1}}{\lambda-\alpha \frac{1+\psi}{\psi+\alpha}} .
$$

Defining $\varphi=\frac{1+\psi}{(1-\alpha) \psi+(1+\alpha \psi) \eta_{c}}$ and recognize that

$$
\begin{gathered}
e^{\frac{1}{2} \varphi^{2} \tau_{\varepsilon}^{-1} \frac{\Phi\left(\varphi \tau_{\varepsilon}^{-1 / 2}+\frac{A-A^{*}}{\tau_{\varepsilon}^{-1 / 2}}\right)}{\Phi\left(\sqrt{\tau_{\varepsilon}}\left(A-A^{*}\right)\right)}}=E\left[e^{\varphi\left(A_{j}-A\right)} \mid A, A_{j} \geq A^{*}\right], \\
e^{\frac{1}{2}\left(1-\eta_{c}\right)^{2} \varphi^{2} \tau_{\varepsilon}^{-1}} \frac{\Phi\left(\left(1-\eta_{c}\right) \varphi \tau_{\varepsilon}^{-1 / 2}+\frac{A-A^{*}}{\tau_{\varepsilon}^{-1 / 2}}\right)}{\Phi\left(\sqrt{\tau_{\varepsilon}}\left(A-A^{*}\right)\right)}=E\left[e^{\left(1-\eta_{c}\right) \varphi\left(A_{j}-A\right)} \mid A, A_{j} \geq A^{*}\right] .
\end{gathered}
$$

we arrive at the expressions in the statement of the proposition. 


\section{A.3 Proof of Proposition 3}

We now derive household $i$ 's optimal cutoff, given that other households all use an equilibrium cutoff $A^{*}$. By substituting for prices, the optimal labor and capital choices of household $i$, the realized capital price $R$, and capital demand $K_{i}$ from Proposition 2 , the utility of household $i$ at $t=1$ from choosing to live in the neighborhood is

$$
\begin{aligned}
E\left[U_{i} \mid \mathcal{I}_{i}\right]= & (1-\alpha) \frac{\psi}{1+\psi} e^{u_{0}+u_{A} A+\frac{(1+\psi)\left(1-\eta_{c}\right)}{(1-\alpha) \psi+(1+\alpha) \eta_{c}}} A_{i}\left(\frac{\Phi\left(\frac{1+\psi}{(1-\alpha) \psi+(1+\alpha \psi) \eta_{c}} \tau_{\varepsilon}^{-1 / 2}+\frac{A-A^{*}}{\tau_{\varepsilon}^{-1 / 2}}\right)}{\Phi\left(\sqrt{\tau_{\varepsilon}}\left(A-A^{*}\right)\right)}\right)^{u_{\Phi}} \\
& \times\left(\frac{\Phi\left(\frac{(1+\psi)\left(1-\eta_{c}\right)}{(1-\alpha) \psi+(1+\alpha \psi) \eta_{c}} \tau_{\varepsilon}^{-1 / 2}+\frac{A-A^{*}}{\tau_{\varepsilon}^{-1 / 2}}\right)}{\Phi\left(\sqrt{\tau_{\varepsilon}}\left(A-A^{*}\right)\right)}\right)
\end{aligned}
$$

where

$$
\begin{aligned}
u_{0}= & \frac{1+\psi}{2(1-\alpha) \psi}\left(\lambda \eta_{c} \frac{1-\alpha \frac{1+\psi}{\psi+\alpha}}{\lambda-\alpha \frac{1+\psi}{\psi+\alpha}}-\frac{\alpha(\lambda-1)}{\lambda-\alpha \frac{1+\psi}{\psi+\alpha}} \frac{\psi(1-\alpha)}{\psi+\alpha}\left(1-\eta_{c}\right)^{2}\right)\left(\frac{1+\psi}{(1-\alpha) \psi+(1+\alpha \psi) \eta_{c}}\right)^{2} \tau_{\varepsilon}^{-1} \\
& +\frac{1}{\psi} \frac{1-\alpha \frac{1+\psi}{\psi+\alpha}}{\lambda-\alpha \frac{1+\psi}{\psi+\alpha}}\left(\frac{\alpha}{1-\alpha}(1+\psi) \log \alpha+\lambda \log (1-\alpha)\right), \\
u_{A}= & \frac{1}{1-\alpha} \frac{1+\psi}{\psi}\left(\frac{1+\psi}{(1-\alpha) \psi+(1+\alpha \psi) \eta_{c}} \eta_{c}-(\lambda-1) \frac{\alpha \frac{1+\psi}{\psi+\alpha}}{\lambda-\alpha \frac{1+\psi}{\psi+\alpha}}\right), \\
u_{\Phi}= & \frac{\lambda \frac{1+\psi}{\psi+\alpha}}{\lambda-\alpha \frac{1+\psi}{\psi+\alpha}} \eta_{c}>0 .
\end{aligned}
$$

Since the household with the critical productivity $A^{*}$ must be indifferent to its moving decision at the cutoff, it follows that $U_{i}-P=0$, which implies

$$
\begin{aligned}
& e^{\frac{(1+\psi)\left(1-\eta_{c}\right)}{(1-\alpha) \psi+(1+\alpha \psi) \eta_{c}}} A_{i}\left(\frac{\Phi\left(\frac{(1+\psi) \tau_{\varepsilon}^{-1 / 2}}{(1-\alpha) \psi+(1+\alpha \psi) \eta_{c}}+\frac{A-A^{*}}{\tau_{\varepsilon}^{-1 / 2}}\right)}{\Phi\left(\sqrt{\tau_{\varepsilon}}\left(A-A^{*}\right)\right)}\right)^{u_{\Phi}}\left(\frac{\Phi\left(\frac{(1+\psi)\left(1-\eta_{c}\right) \tau_{\varepsilon}^{-1 / 2}}{(1-\alpha) \psi+(1+\alpha \psi) \eta_{c}}+\frac{A-A^{*}}{\tau_{\varepsilon}^{-1 / 2}}\right)}{\Phi\left(\sqrt{\tau_{\varepsilon}}\left(A-A^{*}\right)\right)}\right)^{\frac{(1-\lambda) \alpha \frac{1+\psi}{\psi+\alpha}}{\lambda-\alpha \frac{1+\psi}{\psi+\alpha}}} \\
= & \frac{1+\psi}{\psi(1-\alpha)} e^{-u_{0}-u_{A} A} P, \text { with } A_{i}=A^{*}
\end{aligned}
$$

which implies the benefit of living with more productive households is offset by the higher cost of living in the neighborhood.

Fixing the critical value $A^{*}$ and price $P$, we see that the LHS of equation (20) is increasing in monotonically in $A_{i}$, since $\frac{1+\psi}{(1-\alpha) \psi+(1+\alpha \psi) \eta_{c}}\left(1-\eta_{c}\right)>0$. This confirms the optimality of the cutoff strategy that households with $A_{i} \geq A^{*}$ enter the neighborhood, and households with $A_{i}<A^{*}$ choose to live somewhere else. Since $A_{i}=A+\varepsilon_{i}$, it then follows that a fraction 
$\Phi\left(-\sqrt{\tau_{\varepsilon}}\left(A^{*}-A\right)\right)$ enter the neighborhood, and a fraction $\Phi\left(\sqrt{\tau_{\varepsilon}}\left(A^{*}-A\right)\right)$ choose to live somewhere else. As one can see, it is the integral over the idiosyncratic productivity shocks of households $\varepsilon_{i}$ that determines the fraction of households in the neighborhood.

From the optimal supply of housing by builder $i$ in the neighborhood (6), there exists a critical value $\omega^{*}$ :

$$
\omega^{*}=-(1+k) \log P,
$$

such that builders with productivity $\omega_{i} \geq \omega^{*}$ build houses. Thus, a fraction $\Phi\left(-\sqrt{\tau_{e}}\left(\omega^{*}-\xi\right)\right)$ build houses in the neighborhood. Imposing market-clearing, it must be the case that

$$
\Phi\left(-\sqrt{\tau_{\varepsilon}}\left(A^{*}-A\right)\right)=\Phi\left(-\sqrt{\tau_{e}}\left(\omega^{*}-\xi\right)\right) .
$$

Since the CDF of the normal distribution is monotonically increasing, we can invert the above market-clearing conditions, and impose equation (21) to arrive at

$$
\log P=\frac{1}{1+k}\left(\sqrt{\frac{\tau_{\varepsilon}}{\tau_{e}}}\left(A-A^{*}\right)-\xi\right) .
$$

By substituting for $P$ in equation (20), we obtain an equation to determine the equilibrium cutoff $A^{*}=A^{*}(A, \xi)$

$$
\begin{aligned}
& e^{\left(\frac{(1+\psi)\left(1-\eta_{c}\right)}{(1-\alpha) \psi+(1+\alpha \psi) \eta_{c}}+\frac{\sqrt{\tau_{\varepsilon} / \tau_{e}}}{1+k}\right) A^{*}}\left(\frac{\Phi\left(\frac{(1+\psi)\left(1-\eta_{c}\right) \tau_{\varepsilon}^{-1 / 2}}{(1-\alpha) \psi+(1+\alpha \psi) \eta_{c}}+\frac{A-A^{*}}{\tau_{\varepsilon}^{-1 / 2}}\right)}{\Phi\left(\frac{A-A^{*}}{\tau_{\varepsilon}^{-1 / 2}}\right)}\right)^{\frac{(1-\lambda) \alpha \frac{1+\psi}{\psi+\alpha}}{\lambda-\alpha \frac{1+\psi}{\psi+\alpha}}} \frac{\Phi\left(\frac{(1+\psi) \tau_{\varepsilon}^{-1 / 2}}{(1-\alpha) \psi+(1+\alpha \psi) \eta_{c}}+\frac{A-A^{*}}{\tau_{\varepsilon}^{-1 / 2}}\right)^{u_{\Phi}}}{\Phi\left(\frac{A-A^{*}}{\tau_{\varepsilon}^{-1 / 2}}\right)^{u_{\Phi}}} \\
& =\frac{1+\psi}{\psi(1-\alpha)} e^{\left(\frac{\sqrt{\tau_{\varepsilon} / \tau_{e}}}{1+k}-u_{A}\right) A-\frac{1}{1+k} \xi-u_{0}} .
\end{aligned}
$$

Taking the derivative of the log of the LHS of equation (23) with respect to $A^{*}$ gives

$$
\begin{aligned}
& \frac{d \log L H S}{d A^{*}} \\
= & u_{\Phi} \frac{1}{\tau_{\varepsilon}^{-1 / 2}}\left(\frac{\phi\left(\frac{A-A^{*}}{\tau_{\varepsilon}^{-1 / 2}}\right)}{\Phi\left(\frac{A-A^{*}}{\tau_{\varepsilon}^{-1 / 2}}\right)}-\frac{\phi\left(\frac{1+\psi}{(1-\alpha) \psi+(1+\alpha \psi) \eta_{c}} \tau_{\varepsilon}^{-1 / 2}+\frac{A-A^{*}}{\tau_{\varepsilon}^{-1 / 2}}\right)}{\Phi\left(\frac{1+\psi}{(1-\alpha) \psi+(1+\alpha \psi) \eta_{c}} \tau_{\varepsilon}^{-1 / 2}+\frac{A-A^{*}}{\tau_{\varepsilon}^{-1 / 2}}\right)}\right)+\frac{(1+\psi)\left(1-\eta_{c}\right)}{(1-\alpha) \psi+(1+\alpha \psi) \eta_{c}} \\
& +\frac{1}{1+k} \sqrt{\frac{\tau_{\varepsilon}}{\tau_{e}}}-\frac{1}{\tau_{\varepsilon}^{-1 / 2}} \frac{(\lambda-1) \alpha \frac{1+\psi}{\psi+\alpha}}{\lambda-\alpha \frac{1+\psi}{\psi+\alpha}}\left(\frac{\phi\left(\frac{A-A^{*}}{\tau_{\varepsilon}^{-1 / 2}}\right)}{\Phi\left(\frac{A-A^{*}}{\tau_{\varepsilon}^{-1 / 2}}\right)}-\frac{\phi\left(\frac{(1+\psi)\left(1-\eta_{c}\right)}{(1-\alpha) \psi+(1+\alpha \psi) \eta_{c}} \tau_{\varepsilon}^{-1 / 2}+\frac{A-A^{*}}{\tau_{\varepsilon}^{-1 / 2}}\right)}{\Phi\left(\frac{(1+\psi)\left(1-\eta_{c}\right)}{(1-\alpha) \psi+(1+\alpha \psi) \eta_{c}} \tau_{\varepsilon}^{-1 / 2}+\frac{A-A^{*}}{\tau_{\varepsilon}^{-1 / 2}}\right)}\right) .
\end{aligned}
$$

The term in parentheses are nonnegative by the properties of the normal CDF. The last term is nonpositive, since $\lambda>1$, and attains its minimum at $A^{*} \rightarrow \infty$, from which follows, substituting for $u_{\Phi}$, that

$$
\text { as } A^{*} \rightarrow \infty, \frac{d \log L H S}{d A^{*}} \rightarrow \frac{1}{1+k} \sqrt{\frac{\tau_{\varepsilon}}{\tau_{e}}}+\frac{\lambda \frac{1+\psi}{\psi+\alpha}}{\lambda-\alpha \frac{1+\psi}{\psi+\alpha}}>0 \text {. }
$$


Consequently, since $\frac{d \log L H S}{d A^{*}}>0$ when the last term attains its (nonpositive) minimum, it follows that $\frac{d \log L H S}{d A^{*}}>0$. Therefore, $\log L H S$, and consequently $L H S$, is monotonically increasing in $A^{*}$. Since the RHS of equation (23) is independent of $A^{*}$, it follows that the LHS and RHS of equation (23) intersect at most once. Therefore, the can be, at most, one cutoff equilibrium. Furthermore, since the LHS of equation (23) tends to 0 as $A^{*} \rightarrow-\infty$, and the RHS is nonnegative, it follows that a cutoff equilibrium always exists. Therefore, there exists a unique cutoff equilibrium in this economy.

It is straightforward to apply the Implicit Function Theorem to (23) to obtain

$$
\begin{aligned}
\frac{d A^{*}}{d A} & =\frac{\frac{1}{1+k} \sqrt{\frac{\tau_{\varepsilon}}{\tau_{e}}}-\frac{d \log L H S}{d A}-u_{A}}{\frac{d \log L H S}{d A^{*}}} \\
\frac{d A^{*}}{d \xi} & =-\frac{1}{1+k} \frac{1}{\frac{d \log L H S}{d A^{*}}}<0,
\end{aligned}
$$

where

$$
\begin{aligned}
\frac{d \log L H S}{d A}= & -u_{\Phi} \frac{1}{\tau_{\varepsilon}^{-1 / 2}}\left(\frac{\phi\left(\frac{A-A^{*}}{\tau_{\varepsilon}^{-1 / 2}}\right)}{\Phi\left(\frac{A-A^{*}}{\tau_{\varepsilon}^{-1 / 2}}\right)}-\frac{\phi\left(\frac{1+\psi}{(1-\alpha) \psi+(1+\alpha \psi) \eta_{c}} \tau_{\varepsilon}^{-1 / 2}+\frac{A-A^{*}}{\tau_{\varepsilon}^{-1 / 2}}\right)}{\Phi\left(\frac{1+\psi}{(1-\alpha) \psi+(1+\alpha \psi) \eta_{c}} \tau_{\varepsilon}^{-1 / 2}+\frac{A-A^{*}}{\tau_{\varepsilon}^{-1 / 2}}\right)}\right) \\
& +\frac{1}{\tau_{\varepsilon}^{-1 / 2}}(\lambda-1) \frac{\alpha \frac{1+\psi}{\psi+\alpha}}{\lambda-\alpha \frac{1+\psi}{\psi+\alpha}}\left(\frac{\phi\left(\frac{A-A^{*}}{\tau_{\varepsilon}^{-1 / 2}}\right)}{\Phi\left(\frac{A-A^{*}}{\tau_{\varepsilon}^{-1 / 2}}\right)}-\frac{\phi\left(\frac{(1+\psi)\left(1-\eta_{c}\right)}{(1-\alpha) \psi+(1+\alpha \psi) \eta_{c}} \tau_{\varepsilon}^{-1 / 2}+\frac{A-A^{*}}{\tau_{\varepsilon}^{-1 / 2}}\right)}{\Phi\left(\frac{(1+\psi)\left(1-\eta_{c}\right)}{(1-\alpha) \psi+(1+\alpha \psi) \eta_{c}} \tau_{\varepsilon}^{-1 / 2}+\frac{A-A^{*}}{\tau_{\varepsilon}^{-1 / 2}}\right)}\right) .
\end{aligned}
$$

Note that the nonpositive term in $\frac{d \log L H S}{d A}$ achieves its minimum at $A \rightarrow-\infty$, at which

$$
\frac{d \log L H S}{d A} \rightarrow\left((\lambda-1) \alpha\left(1-\eta_{c}\right)-\lambda \eta_{c}\right) \frac{\frac{1+\psi}{\psi+\alpha}}{\lambda-\alpha \frac{1+\psi}{\psi+\alpha}} \frac{1+\psi}{(1-\alpha) \psi+(1+\alpha \psi) \eta_{c}}
$$

Then, as $A \rightarrow-\infty$, the numerator of $\frac{d A^{*}}{d A}$ converges to

$$
\begin{aligned}
\frac{1}{1+k} \sqrt{\frac{\tau_{\varepsilon}}{\tau_{e}}}-\frac{d \log L H S}{d A}-u_{A} \rightarrow & A \rightarrow-\infty-\frac{(1+\psi)\left(\frac{\left((\lambda-1) \alpha\left(1-\eta_{c}\right)-\lambda \eta_{c}\right) \frac{1+\psi}{\psi+\alpha}}{\lambda-\alpha \frac{1+\psi}{\psi+\alpha}}+\frac{1}{1-\alpha} \frac{1+\psi}{\psi} \eta_{c}\right)}{(1-\alpha) \psi+(1+\alpha \psi) \eta_{c}} \\
& +\frac{1}{1-\alpha} \frac{1+\psi}{\psi} \frac{(\lambda-1) \alpha \frac{1+\psi}{\psi+\alpha}}{\lambda-\alpha \frac{1+\psi}{\psi+\alpha}}+\frac{1}{1+k} \sqrt{\frac{\tau_{\varepsilon}}{\tau_{e}}},
\end{aligned}
$$

which is positive. Consequently $\left.\frac{d A^{*}}{d A}\right|_{A^{*}=-\infty}>0$. In contrast, as $A^{*} \rightarrow \infty$, one has that

$$
\begin{aligned}
& \frac{1}{1+k} \sqrt{\frac{\tau_{\varepsilon}}{\tau_{e}}}-\frac{d \log L H S}{d A}-u_{A} \\
\rightarrow & A \rightarrow \infty \frac{1}{1+k} \sqrt{\frac{\tau_{\varepsilon}}{\tau_{e}}}-\frac{1}{1-\alpha} \frac{1+\psi}{\psi}\left(\frac{1+\psi}{(1-\alpha) \psi+(1+\alpha \psi) \eta_{c}} \eta_{c}-(\lambda-1) \frac{\alpha \frac{1+\psi}{\psi+\alpha}}{\lambda-\alpha \frac{1+\psi}{\psi+\alpha}}\right),
\end{aligned}
$$


which is negative if

$$
\eta_{c}>\eta_{c}^{*}=(1-\alpha) \frac{\psi}{1+\alpha \psi} \frac{\frac{\psi}{1+\psi} \frac{1-\alpha}{1+k} \sqrt{\frac{\tau_{\varepsilon}}{\tau_{e}}}+(\lambda-1) \frac{\alpha \frac{1+\psi}{\psi+\alpha}}{\lambda-\alpha \frac{1+\psi}{\psi+\alpha}}}{\frac{1+\psi}{1+\alpha \psi}-\frac{\psi}{1+\psi} \frac{1-\alpha}{1+k} \sqrt{\frac{\tau_{\varepsilon}}{\tau_{e}}}-(\lambda-1) \frac{\alpha \frac{1+\psi}{\psi+\alpha}}{\lambda-\alpha \frac{1+\psi}{\psi+\alpha}}} .
$$

We can rewrite equation (23) as

$$
\begin{aligned}
& \left.e^{-\left(\frac{(1+\psi)\left(1-\eta_{c}\right)}{(1-\alpha) \psi+(1+\alpha \psi) \eta_{c}}+\frac{1}{1+k} \sqrt{\frac{\tau_{\varepsilon}}{\tau_{e}}}\right) s\left(\frac{\Phi\left(\frac{(1+\psi)\left(1-\eta_{c}\right) \tau_{\varepsilon}^{-1 / 2}}{(1-\alpha) \psi+(1+\alpha \psi) \eta_{c}}+\frac{s}{\tau_{\varepsilon}^{-1 / 2}}\right)}{\Phi\left(\frac{s}{\tau_{\varepsilon}^{-1 / 2}}\right)}\right)}\right)^{\frac{(1-\lambda) \alpha \frac{1+\psi}{\psi+\alpha}}{\lambda-\alpha \frac{1+\psi}{\psi+\alpha}}} \frac{\Phi\left(\frac{(1+\psi) \tau_{\varepsilon}^{-1 / 2}}{(1-\alpha) \psi+(1+\alpha \psi) \eta_{c}}+\frac{s}{\tau_{\varepsilon}^{-1 / 2}}\right)^{u_{\Phi}}}{\Phi\left(\frac{s}{\tau_{\varepsilon}^{-1 / 2}}\right)^{u_{\Phi}}} \\
& =\frac{1+\psi}{\psi(1-\alpha)} e^{-\lambda \frac{1}{1-\alpha} \frac{1+\psi}{\psi} \frac{1-\alpha \frac{1+\psi}{\psi+\alpha}}{\lambda-\alpha \frac{1+\psi}{\psi+\alpha}} A-\frac{1}{1+k} \xi-u_{0}}
\end{aligned}
$$

where $s=A-A^{*}$ determines the population that enter the neighborhood. It is straightforward to show that

$$
\frac{d \log L H S}{d s}=-\frac{d \log L H S}{d A^{*}}<0
$$

Consequently, we have

$$
\begin{aligned}
\frac{d s}{d \xi} & =-\frac{\frac{1}{1+k}}{\frac{d \log L H S}{d s}}>0, \\
\frac{d s}{d A} & =-\frac{\lambda \frac{1}{1-\alpha} \frac{1+\psi}{\psi} \frac{1-\alpha \frac{1+\psi}{\psi+\alpha}}{\lambda-\alpha \frac{1+\psi}{\psi+\alpha}}}{\frac{d \log L H S}{d s}}>0 .
\end{aligned}
$$

Thus, the population that enters, $\Phi\left(\sqrt{\tau_{\varepsilon}} s\right)$, is increasing in $A$ and $\xi$. Furthermore, it follows from (22) that

$$
\frac{d \log P}{d A}=\frac{1}{1+k} \sqrt{\frac{\tau_{\varepsilon}}{\tau_{e}}} \frac{d s}{d A}>0,
$$

and therefore the log housing price is increasing in $A$.

Finally, we recognize that

$$
\frac{d^{2} P}{d A^{2}}=\left(\frac{d s}{d A}\right)^{2} P+\frac{d^{2} s}{d A^{2}} P=\left(\frac{d s}{d A}\right)^{2} P+\frac{\lambda \frac{1}{1-\alpha} \frac{1+\psi}{\psi} \frac{1-\alpha \frac{1+\psi}{\psi+\alpha}}{\lambda-\alpha \frac{1+\psi}{\psi+\alpha}}}{\left(\frac{d \log L H S}{d s}\right)^{2}} \frac{d s}{d A} \frac{d^{2} \log L H S}{d s^{2}} P
$$

where $\lambda \frac{1}{1-\alpha} \frac{1+\psi}{\psi} \frac{1-\alpha \frac{1+\psi}{\psi+\alpha}}{\lambda-\alpha \frac{1+\psi}{\psi+\alpha}} \frac{d s}{d A}>0$ by the above arguments. It follows that from calculating $\frac{d^{2} \log L H S}{d s^{2}}$ that

$$
\lim _{s \rightarrow-\infty} \frac{d^{2} \log L H S}{d s^{2}}=\left(\lambda\left(\alpha-\eta_{c}\right)-\alpha\right) \frac{\frac{1+\psi}{\psi+\alpha}}{\lambda-\alpha \frac{1+\psi}{\psi+\alpha}} \frac{1}{\tau_{\varepsilon}^{-1}}
$$


and therefore, as $P \rightarrow \infty$, from the expression for $\frac{d^{2} P}{d A^{2}}$ one has that $\frac{d^{2} P}{d A^{2}} \rightarrow \infty$. Furthermore, as $s \rightarrow-\infty$,

$$
\frac{d \log L H S}{d s} \rightarrow-\left(\frac{1}{1+k} \sqrt{\frac{\tau_{\varepsilon}}{\tau_{e}}}+\frac{\lambda \frac{1+\psi}{\psi+\alpha}}{\lambda-\alpha \frac{1+\psi}{\psi+\alpha}}\right)
$$

and

$$
\lim _{s \rightarrow \infty} \frac{d^{2} \log L H S}{d s^{2}}=0
$$

and $P \rightarrow 0$ at an exponential rate. Consequently, as $s \rightarrow-\infty, \frac{d^{2} P}{d A^{2}} \rightarrow 0$. Since $\frac{d^{2} P}{d A^{2}}$ is continuous, it follows that $\frac{d^{2} P}{d A^{2}} \geq 0$. Consequently, $P$ is convex in $A$. Since, in equilibrium, the housing price is equal to the utility of the household with the cutoff productivity, it follows that this utility is also convex and increasing in $A$.

\section{A.4 Proof of Proposition 4}

Given our assumption about the sufficient statistic in housing price, each household's posterior about $A$ is Gaussian $A \mid \mathcal{I}_{i} \sim \mathcal{N}\left(\hat{A}_{i}, \hat{\tau}_{A}^{-1}\right)$ with conditional mean and variance of

$$
\begin{aligned}
\hat{A}_{i} & =\bar{A}+\tau_{A}^{-1}\left[\begin{array}{lll}
1 & 1 & 1
\end{array}\right]\left[\begin{array}{ccc}
\tau_{A}^{-1}+\tau_{Q}^{-1} & \tau_{A}^{-1} & \tau_{A}^{-1} \\
\tau_{A}^{-1} & \tau_{A}^{-1}+z_{\xi}^{-2} \tau_{\xi}^{-1} & \tau_{A}^{-1} \\
\tau_{A}^{-1} & \tau_{A}^{-1} & \tau_{A}^{-1}+\tau_{\varepsilon}^{-1}
\end{array}\right]^{-1}\left[\begin{array}{c}
Q-\bar{A} \\
z(P)-\bar{A} \\
A_{i}-\bar{A}
\end{array}\right] \\
& =\hat{\tau}_{A}^{-1}\left(\tau_{A} \bar{A}+\tau_{Q} Q+z_{\xi}^{2} \tau_{\xi} z(P)+\tau_{\varepsilon} A_{i}\right), \\
\hat{\tau}_{A} & =\tau_{A}+\tau_{Q}+z_{\xi}^{2} \tau_{\xi}+\tau_{\varepsilon} .
\end{aligned}
$$

Note that the conditional estimate of $\hat{A}_{i}$ of household $i$ is increasing in its own productivity $A_{i}$. Similarly, the posterior for capital producers about $A$ is Gaussian $A \mid \mathcal{I}^{c} \sim \mathcal{N}\left(\hat{A}^{c}, \hat{\tau}_{A}^{c-1}\right)$, where

$$
\begin{aligned}
\hat{A}^{c} & =\bar{A}+\tau_{A}^{-1}\left[\begin{array}{ll}
1 & 1
\end{array}\right]\left[\begin{array}{cc}
\tau_{A}^{-1}+\tau_{Q}^{-1} & \tau_{A}^{-1} \\
\tau_{A}^{-1} & \tau_{A}^{-1}+z_{\xi}^{-2} \tau_{\xi}^{-1}
\end{array}\right]^{-1}\left[\begin{array}{c}
Q-\bar{A} \\
z(P)-\bar{A}
\end{array}\right] \\
& =\hat{\tau}_{A}^{c-1}\left(\tau_{A} \bar{A}+\tau_{Q} Q+z_{\xi}^{2} \tau_{\xi} z(P)\right), \\
\hat{\tau}_{A}^{c} & =\tau_{A}+\tau_{Q}+z_{\xi}^{2} \tau_{\xi} .
\end{aligned}
$$

This completes our characterization of learning by households and capital producers.

We now turn to the optimal decision of capital producers. Since the posterior for $A-A^{*}$ of households is conditionally Gaussian, it follows that the expectations in the expression of $K$ in Proposition 2 is a function of the two conditional moments, $\hat{A}^{c}-A^{*}$ and $\hat{\tau}_{A}^{c}$. Let

$\left.F\left(\hat{A}^{c}-A^{*}, \hat{\tau}_{A}^{c}\right)=E\left[\left(\frac{e^{\left(A-A^{*}\right)} \Phi\left(\frac{1+\psi}{(1-\alpha) \psi+(1+\alpha \psi) \eta_{c}} \tau_{\varepsilon}^{-1 / 2}+\frac{A-A^{*}}{\tau_{\varepsilon}^{-1 / 2}}\right)^{\eta_{c}}}{\Phi\left(\frac{A-A^{*}}{\tau_{\varepsilon}^{-1 / 2}}\right)^{\eta_{c}+\frac{\psi(1-\alpha)}{1+\psi}} \Phi\left(\frac{(1+\psi)\left(1-\eta_{c}\right)}{(1-\alpha) \psi+(1+\alpha \psi) \eta_{c}} \tau_{\varepsilon}^{-1 / 2}+\frac{A-A^{*}}{\tau_{\varepsilon}^{-1 / 2}}\right)^{-\frac{\psi(1-\alpha)}{1+\psi}}}\right)^{\frac{1+\psi}{\psi+\alpha}}\right] \mathcal{I}^{c}\right]$. 
Define $z=\frac{A-A^{*}}{\tau_{\varepsilon}^{-1 / 2}}$ and the function $f(z)$

$$
f(z)=e^{\tau_{\varepsilon}^{-1 / 2}} z \frac{\Phi\left(\frac{1+\psi}{(1-\alpha) \psi+(1+\alpha \psi) \eta_{c}} \tau_{\varepsilon}^{-1 / 2}+z\right)^{\eta_{c}}}{\Phi(z)^{\eta_{c}}}\left(\frac{\Phi\left(\frac{(1+\psi)\left(1-\eta_{c}\right)}{(1-\alpha) \psi+(1+\alpha \psi) \eta_{c}} \tau_{\varepsilon}^{-1 / 2}+z\right)}{\Phi(z)}\right)^{\frac{\psi}{1+\psi}(1-\alpha)}
$$

which is the term inside the bracket in the expectation. Then, it follows that

$$
\begin{aligned}
& \frac{1}{f(z)} \frac{d f(z)}{d z}=\tau_{\varepsilon}^{-1 / 2}+\eta_{c}\left(\frac{\phi\left(\frac{1+\psi}{(1-\alpha) \psi+(1+\alpha \psi) \eta_{c}} \tau_{\varepsilon}^{-1 / 2}+z\right)}{\Phi\left(\frac{1+\psi}{(1-\alpha) \psi+(1+\alpha \psi) \eta_{c}} \tau_{\varepsilon}^{-1 / 2}+z\right)}-\frac{\phi(z)}{\Phi(z)}\right) \\
& +\frac{\psi}{1+\psi}(1-\alpha)\left(\frac{\phi\left(\frac{(1+\psi)\left(1-\eta_{c}\right)}{(1-\alpha) \psi+(1+\alpha \psi) \eta_{c}} \tau_{\varepsilon}^{-1 / 2}+z\right)}{\Phi\left(\frac{(1+\psi)\left(1-\eta_{c}\right)}{(1-\alpha) \psi+(1+\alpha \psi) \eta_{c}} \tau_{\varepsilon}^{-1 / 2}+z\right)}-\frac{\phi(z)}{\Phi(z)}\right) \text {. }
\end{aligned}
$$

Notice that $\frac{\phi\left(\frac{1+\psi}{(1-\alpha) \psi+(1+\alpha \psi) \eta_{c}} \tau_{\varepsilon}^{-1 / 2}+z\right)}{\Phi\left(\frac{1+\psi}{(1-\alpha) \psi+(1+\alpha \psi) \eta_{c}} \tau_{\varepsilon}^{-1 / 2}+z\right)}-\frac{\phi(z)}{\Phi(z)}$ achieves its minimum as $z \rightarrow-\infty$. Applying L'Hospital's Rule, it follows that the minimum of $\frac{1}{f(z)} \frac{d f(z)}{d z}$ is given by

$$
\lim _{z \rightarrow-\infty} \frac{1}{f(z)} \frac{d f(z)}{d z}=\alpha \frac{1+\psi}{\psi+\alpha+(1-\alpha) \eta_{c}}\left(1-\eta_{c}\right) \tau_{\varepsilon}^{-1 / 2}>0
$$

from which follows that $\frac{1}{f(z)} \frac{d f(z)}{d z} \geq 0$ for all $z$, and therefore $\frac{d f(z)}{d z} \geq 0$, since $f(z) \geq 0$. Consequently, since $f(z)^{\frac{1+\psi}{\psi+\alpha}}$ is a monotonic transformation of $f(z)$, it follows that $\frac{d F}{d x}\left(x, \hat{\tau}_{A}\right)$ $\geq 0$ since this holds for all realizations of $A-A^{*}$. This establishes that the optimal choice of capital is increasing with $\hat{A}^{c}$, since $f(z)$ is increasing for each realization of $z$.

The optimal choice of $K$ then takes the following form

$$
\log K=\frac{1}{\lambda-\alpha \frac{1+\psi}{\psi+\alpha}} \log F\left(\hat{A}^{c}-A^{*}, \hat{\tau}_{A}^{c}\right)+\frac{\frac{1+\psi}{\psi+\alpha}}{\lambda-\alpha \frac{1+\psi}{\psi+\alpha}} A^{*}+k_{0} .
$$

By substituting the expressions for $K_{i}$ and $l_{i}$ into the utility of household $i$ given in Proposition 1 , we obtain

$$
\begin{aligned}
& E\left[U_{i} \mid \mathcal{I}_{i}\right] \\
& =(1-\alpha) \frac{\psi}{1+\psi} e^{\frac{(1+\psi)\left(1-\eta_{c}\right)}{(1-\alpha) \psi+(1+\alpha \psi) \eta_{c}} A_{i}+\frac{\alpha \frac{1+\psi}{\psi+\alpha}}{\lambda-\alpha \frac{1+\psi}{\psi+\alpha}}\left(\log F\left(\hat{A}^{c}-A^{*}, \hat{\tau}_{A}^{c}\right)+\frac{1+\psi}{\psi+\alpha} A^{*}\right)+\frac{1}{1-\alpha} \frac{1+\psi}{\psi}\left(\frac{1+\psi}{(1-\alpha) \psi+(1+\alpha \psi) \eta_{c}} \eta_{c}-\alpha \frac{1+\psi}{\psi+\alpha}\right) A^{*}+u_{0}} \\
& \cdot E\left[\left(\frac{e^{\frac{1}{1-\alpha} \frac{\psi+\alpha}{\psi}\left(\frac{(1+\psi) \eta_{c}}{(1-\alpha) \psi+(1+\alpha \psi) \eta_{c}}-\alpha \frac{1+\psi}{\psi+\alpha}\right)\left(A-A^{*}\right)} \Phi\left(\frac{(1+\psi) \tau_{\varepsilon}^{-1 / 2}}{(1-\alpha) \psi+(1+\alpha \psi) \eta_{c}}+\frac{A-A^{*}}{\tau_{\varepsilon}^{-1 / 2}}\right)^{\eta_{c}}}{\Phi\left(\frac{A-A^{*}}{\tau_{\varepsilon}^{-1 / 2}}\right)^{\eta_{c}-\alpha} \Phi\left(\frac{(1+\psi)\left(1-\eta_{c}\right) \tau_{\varepsilon}^{-1 / 2}}{(1-\alpha) \psi+(1+\alpha \psi) \eta_{c}}+\frac{A-A^{*}}{\tau_{\varepsilon}^{-1 / 2}}\right)^{\frac{1+\psi}{\psi+\alpha}}}\right)^{\mathcal{I}_{i}}\right]
\end{aligned}
$$

where $u_{0}$ is given in the proof of Proposition 3. When $A_{i}=A^{*}$, this further reduces to 


$$
\begin{aligned}
& E\left[U_{i} \mid \mathcal{I}_{i}\right] \\
= & (1-\alpha) \frac{\psi}{1+\psi} e^{\frac{\lambda \frac{1+\psi}{\psi+\alpha}}{\lambda-\alpha \frac{1+\psi}{\psi+\alpha}} A^{*}+\frac{\alpha \frac{1+\psi}{\psi+\alpha}}{\lambda-\alpha \frac{1+\psi}{\psi+\alpha}} \log F\left(\hat{A}^{c}-A^{*}, \hat{\tau}_{A}^{c}\right)+u_{0}} \\
\cdot E\left[\left(\frac{e^{\frac{1}{1-\alpha} \frac{\psi+\alpha}{\psi}\left(\frac{(1+\psi) \eta_{c}}{(1-\alpha) \psi+(1+\alpha \psi) \eta_{c}}-\alpha \frac{1+\psi}{\psi+\alpha}\right)\left(A-A^{*}\right)} \Phi\left(\frac{(1+\psi) \tau_{\varepsilon}^{-1 / 2}}{(1-\alpha) \psi+(1+\alpha \psi) \eta_{c}}+\frac{A-A^{*}}{\tau_{\varepsilon}^{-1 / 2}}\right)^{\eta_{c}}}{\Phi\left(\frac{A-A^{*}}{\tau_{\varepsilon}^{-1 / 2}}\right)^{\eta_{c}-\alpha} \Phi\left(\frac{(1+\psi)\left(1-\eta_{c}\right) \tau_{\varepsilon}^{-1 / 2}}{(1-\alpha) \psi+(1+\alpha \psi) \eta_{c}}+\frac{A-A^{*}}{\tau_{\varepsilon}^{-1 / 2}}\right)^{\alpha}}\right)\right] & \left.\mathcal{I}_{i}\right],
\end{aligned}
$$

Since the posterior for $A-A^{*}$ of household $i$ is conditionally Gaussian, it follows that the expectations in the expressions above are functions of the first two conditional moments $\hat{A}_{i}-A^{*}$ and $\hat{\tau}_{A}$. Let

$$
\left.G\left(\hat{A}_{i}-A^{*}, \hat{\tau}_{A}\right)=E\left[\left(\frac{e^{\frac{1}{1-\alpha} \frac{\psi+\alpha}{\psi}\left(\frac{(1+\psi) \eta_{c}}{(1-\alpha) \psi+(1+\alpha \psi) \eta_{c}}-\alpha \frac{1+\psi}{\psi+\alpha}\right)\left(A-A^{*}\right)} \Phi\left(\frac{(1+\psi) \tau_{\varepsilon}^{-1 / 2}}{(1-\alpha) \psi+(1+\alpha \psi) \eta_{c}}+\frac{A-A^{*}}{\tau_{\varepsilon}^{-1 / 2}}\right)^{\eta_{c}}}{\Phi\left(\frac{A-A^{*}}{\tau_{\varepsilon}^{-1 / 2}}\right)^{\eta_{c}-\alpha} \Phi\left(\frac{1+\psi}{\psi+\alpha}\right.}\right)^{\alpha}\right] \mathcal{I}_{i}\right]
$$

Define $z=\frac{A-A^{*}}{\tau_{\varepsilon}^{-1 / 2}}$, and

$$
\begin{aligned}
g(z)= & e^{\frac{1}{1-\alpha} \frac{\psi+\alpha}{\psi}\left(\frac{(1+\psi) \eta_{c}}{(1-\alpha) \psi+(1+\alpha \psi) \eta_{c}}-\alpha \frac{1+\psi}{\psi+\alpha}\right) \tau_{\varepsilon}^{-1 / 2} z} \frac{\Phi\left(\frac{1+\psi}{(1-\alpha) \psi+(1+\alpha \psi) \eta_{c}} \tau_{\varepsilon}^{-1 / 2}+z\right)^{\eta_{c}}}{\Phi(z)^{\eta_{c}}} \\
& \cdot \frac{\Phi\left(\frac{(1+\psi)\left(1-\eta_{c}\right)}{(1-\alpha) \psi+(1+\alpha \psi) \eta_{c}} \tau_{\varepsilon}^{-1 / 2}+z\right)^{-\alpha}}{\Phi(z)^{-\alpha}},
\end{aligned}
$$

as the term inside the bracket. Then, it follows that

$$
\begin{aligned}
\frac{1}{g(z)} \frac{d g(z)}{d z}= & \frac{1}{1-\alpha} \frac{\psi+\alpha}{\psi}\left(\frac{1+\psi}{(1-\alpha) \psi+(1+\alpha \psi) \eta_{c}} \eta_{c}-\alpha \frac{1+\psi}{\psi+\alpha}\right) \tau_{\varepsilon}^{-1 / 2}+\left(\alpha-\eta_{c}\right) \frac{\phi(z)}{\Phi(z)} \\
& +\eta_{c} \frac{\phi\left(\frac{1+\psi}{(1-\alpha) \psi+(1+\alpha \psi) \eta_{c}} \tau_{\varepsilon}^{-1 / 2}+z\right)}{\Phi\left(\frac{1+\psi}{(1-\alpha) \psi+(1+\alpha \psi) \eta_{c}} \tau_{\varepsilon}^{-1 / 2}+z\right)}-\alpha \frac{\phi\left(\frac{(1+\psi)\left(1-\eta_{c}\right)}{(1-\alpha) \psi+(1+\alpha \psi) \eta_{c}} \tau_{\varepsilon}^{-1 / 2}+z\right)}{\Phi\left(\frac{(1+\psi)\left(1-\eta_{c}\right)}{(1-\alpha) \psi+(1+\alpha \psi) \eta_{c}} \tau_{\varepsilon}^{-1 / 2}+z\right)} .
\end{aligned}
$$

Note $\frac{\phi\left(\frac{1+\psi}{\Phi(1-\alpha) \psi+(1+\alpha \psi) \eta_{c}} \tau_{\varepsilon}^{-1 / 2}+z\right)}{\Phi\left(\frac{1+\psi}{(1-\alpha) \psi+(1+\alpha \psi) \eta_{c}} \tau_{\varepsilon}^{-1 / 2}+z\right)}-\frac{\phi(z)}{\Phi(z)}$ achieves its minimum as $z \rightarrow-\infty$. Applying L'Hospital's Rule, it follows, with some manipulation, that the minimum of $\frac{1}{g(z)} \frac{d g(z)}{d z}$ is given by

$$
\lim _{z \rightarrow-\infty} \frac{1}{g(z)} \frac{d g(z)}{d z}=0
$$

It follows that $\frac{1}{g(z)} \frac{d g(z)}{d z} \geq 0$, and therefore $\frac{d g(z)}{d z} \geq 0$, since $g(z) \geq 0$. Consequently, since $g(z)^{\frac{1+\psi}{\psi+\alpha}}$ is a monotonic transformation of $g(z)$, it follows that $\frac{d G}{d x}\left(x, \hat{\tau}_{A}\right) \geq 0$, since this holds for all realizations of $A-A^{*}$. 
Since the household with the critical productivity $A^{*}$ must be indifferent to its moving decision at the cutoff, it follows that $U_{i}-P=0$, which implies

$$
\begin{aligned}
& e^{\frac{(1+\psi)\left(1-\eta_{c}\right)}{(1-\alpha) \psi+(1+\alpha \psi) \eta_{c}} A_{i}+\frac{\alpha \frac{1+\psi}{\psi+\alpha}}{\lambda-\alpha \frac{1+\psi}{\psi+\alpha}}\left(\log F\left(\hat{A}^{c}-A^{*}, \hat{\tau}_{A}^{c}\right)+\frac{1+\psi}{\psi+\alpha} A^{*}\right)+\frac{1}{1-\alpha} \frac{1+\psi}{\psi}\left(\frac{(1+\psi) \eta_{c}}{(1-\alpha) \psi+(1+\alpha \psi) \eta_{c}}-\alpha \frac{1+\psi}{\psi+\alpha}\right) A^{*}+u_{0}} \\
& \cdot G\left(\hat{A}_{i}-A^{*}, \hat{\tau}_{A}\right)=\frac{1+\psi}{\psi(1-\alpha)} P, A_{i}=A^{*}
\end{aligned}
$$

which does not depend on the unobserved $A$ or the supply shock $\xi$. As such, $A^{*}=A^{*}(\log P, Q)$. Furthermore, since $\hat{A}_{i}^{*}$ is increasing in $A_{i}$ and $G\left(\hat{A}_{i}^{*}-A^{*}, \tau_{A}\right)$ is (weakly) increasing in $\hat{A}_{i}$, it follows that the LHS of equation (25) is (weakly) monotonically increasing in $A_{i}$, confirming the cutoff strategy assumed for households is optimal. Those with the RHS being nonnegative enter the neighborhood, and those with it being negative choose to live elsewhere.

It then follows from market-clearing that

$$
\Phi\left(-\sqrt{\tau_{\varepsilon}}\left(A^{*}-A\right)\right)=\Phi\left(-\sqrt{\tau_{e}}\left(\omega^{*}-\xi\right)\right) .
$$

Since the CDF of the normal distribution is monotonically increasing, we can invert the above market-clearing condition, and impose equation (21) to arrive at

$$
\log P=\frac{1}{1+k}\left(\sqrt{\frac{\tau_{\varepsilon}}{\tau_{e}}}\left(A-A^{*}\right)-\xi\right),
$$

from which follows that

$$
z(P)=\sqrt{\frac{\tau_{e}}{\tau_{\varepsilon}}}((1+k) \log P+\bar{\xi})+A^{*}=A-\sqrt{\frac{\tau_{e}}{\tau_{\varepsilon}}}(\xi-\bar{\xi}),
$$

and therefore $z_{\xi}=\sqrt{\frac{T_{\varepsilon}}{\tau_{e}}}$. This confirms our conjecture for the sufficient statistic in housing price and that learning by households is indeed a linear updating rule.

As a consequence, the conditional estimate of household $i$ is

$$
\begin{aligned}
& \hat{A}_{i}=\hat{\tau}_{A}^{-1}\left(\tau_{A} \bar{A}+\tau_{Q} Q+\frac{\tau_{\varepsilon}}{\tau_{e}} \tau_{\xi} z+\tau_{\varepsilon} A_{i}\right), \\
& \hat{\tau}_{A}=\tau_{A}+\tau_{Q}+\frac{\tau_{\varepsilon}}{\tau_{e}} \tau_{\xi}+\tau_{\varepsilon},
\end{aligned}
$$

and the conditional estimate of capital producers is

$$
\begin{aligned}
& \hat{A}^{c}=\hat{\tau}_{A}^{c-1}\left(\tau_{A} \bar{A}+\tau_{Q} Q+\frac{\tau_{\varepsilon}}{\tau_{e}} \tau_{\xi} z\right), \\
& \hat{\tau}_{A}^{c}=\tau_{A}+\tau_{Q}+\frac{\tau_{\varepsilon}}{\tau_{e}} \tau_{\xi} .
\end{aligned}
$$

Substituting for prices, and simplifying $A^{*}$ terms, we can express equation (25) as

$$
e^{\left(\frac{\frac{1+\psi}{\psi+\alpha}}{\lambda-\alpha \frac{1+\psi}{\psi+\alpha}}+\frac{\sqrt{\tau \varepsilon / \tau e}}{1+k}\right) A^{*}} G\left(\hat{A}^{*}-A^{*}, \hat{\tau}_{A}\right) F\left(\hat{A}^{c}-A^{*}, \hat{\tau}_{A}^{c}\right)^{\frac{\alpha \frac{1+\psi}{\psi+\alpha}}{\lambda-\alpha \frac{1+\psi}{\psi+\alpha}}}=\frac{1+\psi}{\psi(1-\alpha)} e^{\frac{1}{1+k} \sqrt{\frac{\tau_{\varepsilon}}{\tau_{e}}} z-\bar{\xi}-u_{0}},
$$


Notice that the LHS of equation (26) is continuous in $A^{*}$. As $A^{*} \rightarrow-\infty$, the LHS of equation (26) converges to

$$
\lim _{A^{* \rightarrow-\infty}} L H S=0 .
$$

Furthermore, by L'Hospital's Rule and the Sandwich Theorem, one also has that

$$
\lim _{A^{*} \rightarrow \infty} L H S=\infty .
$$

Since the RHS is independent of $A^{*}$, it follows that the LHS and RHS intersect once. Therefore, a cutoff equilibrium in the economy with informational frictions exists.

Now consider the derivative of the log of the LHS of equation (26):

$$
\frac{d \log L H S}{d A^{*}}=\frac{\lambda \frac{1+\psi}{\psi+\alpha}}{\lambda-\alpha \frac{1+\psi}{\psi+\alpha}}+\frac{\sqrt{\tau_{\varepsilon} / \tau_{e}}}{1+k}-\frac{\hat{\tau}_{A}^{c}}{\hat{\tau}_{A}} \frac{G^{\prime}\left(\hat{A}^{*}-A^{*}, \hat{\tau}_{A}\right)}{G\left(\hat{A}^{*}-A^{*}, \hat{\tau}_{A}\right)}-\frac{\alpha \frac{1+\psi}{\psi+\alpha}}{\lambda-\alpha \frac{1+\psi}{\psi+\alpha}} \frac{F^{\prime}\left(\hat{A}^{c}-A^{*}, \hat{\tau}_{A}^{c}\right)}{F\left(\hat{A}^{c}-A^{*}, \hat{\tau}_{A}^{c}\right)}
$$

where $G^{\prime}\left(\cdot, \hat{\tau}_{A}\right)$ and $F^{\prime}\left(\cdot, \hat{\tau}_{A}^{c}\right)$ are understood to be first derivatives with respect to the first argument. From our derivation of $\frac{1}{f(z)} \frac{d f(z)}{d z}$ above, we recognize that

$$
\frac{1}{f(z)} \frac{d f(z)}{d z} \leq \tau_{\varepsilon}^{-1 / 2}
$$

since the latter two terms are nonpositive. Furthermore, we can rewrite

$$
\frac{F^{\prime}\left(\hat{A}^{*}-A^{*}, \hat{\tau}_{A}\right)}{F\left(\hat{A}^{*}-A^{*}, \hat{\tau}_{A}\right)}=E\left[\frac{f\left(\sqrt{\tau_{\varepsilon}}\left(A-A^{*}\right)\right)}{F\left(\hat{A}^{*}-A^{*}, \hat{\tau}_{A}\right)}\left(\frac{d \log f(z)}{d z} \underset{z=\sqrt{\tau_{\varepsilon}}\left(A-A^{*}\right)}{ } \sqrt{\tau_{\varepsilon}}\right) \mid \mathcal{I}^{c}\right]
$$

where $E\left[\frac{f\left(\sqrt{\tau_{\varepsilon}}\left(A-A^{*}\right)\right)}{F\left(\hat{A}^{*}-A^{*}, \hat{\tau}_{A}\right)} \mid \mathcal{I}_{i}\right]=1$, so that $w_{a}^{f}=\frac{f\left(\sqrt{\tau_{\varepsilon}}\left(a-A^{*}\right)\right)}{F\left(\hat{A}^{*}-A^{*}, \hat{\tau}_{A}\right)}$ acts as a weighting function. We can take the derivative inside the expectation because $f(z)$ has a continuous first derivative. It then follows that

$$
\frac{F^{\prime}\left(\hat{A}^{*}-A^{*}, \hat{\tau}_{A}\right)}{F\left(\hat{A}^{*}-A^{*}, \hat{\tau}_{A}\right)} \leq \max _{A} \frac{d \log f(z)}{d z} \leq 1
$$

Similarly, recognizing that

$$
\frac{\phi\left(\frac{(1+\psi)\left(1-\eta_{c}\right)}{(1-\alpha) \psi+(1+\alpha \psi) \eta_{c}} \tau_{\varepsilon}^{-1 / 2}+z\right)}{\Phi\left(\frac{(1+\psi)\left(1-\eta_{c}\right)}{(1-\alpha) \psi+(1+\alpha \psi) \eta_{c}} \tau_{\varepsilon}^{-1 / 2}+z\right)}-\frac{\phi\left(\frac{1+\psi}{(1-\alpha) \psi+(1+\alpha \psi) \eta_{c}} \tau_{\varepsilon}^{-1 / 2}+z\right)}{\Phi\left(\frac{1+\psi}{(1-\alpha) \psi+(1+\alpha \psi) \eta_{c}} \tau_{\varepsilon}^{-1 / 2}+z\right)} \geq 0 .
$$

we can bound $\frac{1}{g(z)} \frac{d g(z)}{d z}$ by

$$
\frac{1}{g(z)} \frac{d g(z)}{d z} \leq \frac{1}{1-\alpha} \frac{\psi+\alpha}{\psi}\left(\frac{1+\psi}{(1-\alpha) \psi+(1+\alpha \psi) \eta_{c}} \eta_{c}-\alpha \frac{1+\psi}{\psi+\alpha}\right) \tau_{\varepsilon}^{-1 / 2}
$$


If $\eta_{c} \geq \alpha$, since the latter two terms are always nonpositive, and

$$
\frac{1}{g(z)} \frac{d g(z)}{d z} \leq \frac{1+\psi}{(1-\alpha) \psi+(1+\alpha \psi) \eta_{c}} \alpha \eta_{c} \tau_{\varepsilon}^{-1 / 2}
$$

if $\eta_{c}<\alpha$, since the second term then attains its maximum as $z \rightarrow-\infty$, and we have truncated the third term. Consequently,

$$
\frac{G^{\prime}\left(\hat{A}^{*}-A^{*}, \hat{\tau}_{A}\right)}{G\left(\hat{A}^{*}-A^{*}, \hat{\tau}_{A}\right)} \leq\left\{\begin{array}{c}
\frac{1}{1-\alpha} \frac{\psi+\alpha}{\psi}\left(\frac{1+\psi}{(1-\alpha) \psi+(1+\alpha \psi) \eta_{c}} \eta_{c}-\alpha \frac{1+\psi}{\psi+\alpha}\right), \text { if } \eta_{c} \geq \alpha \\
\frac{1+\psi}{(1-\alpha) \psi+(1+\alpha \psi) \eta_{c}} \alpha \eta_{c}, \text { if } \eta_{c}<\alpha
\end{array} .\right.
$$

If $\eta_{c}<\alpha$, then, since $\frac{\hat{\tau}_{A}^{c}}{\hat{\tau}_{A}} \leq 1$ and $\frac{1+\psi}{\psi+\alpha}>1$, so that $\frac{\lambda-\alpha}{\lambda-\alpha \frac{1+\psi}{\psi+\alpha}} \frac{1+\psi}{\psi+\alpha}>1$, we can bound $\frac{d \log L H S}{d A^{*}}$ from below by

$$
\frac{d \log L H S}{d A^{*}} \geq 1+\frac{\sqrt{\tau_{\varepsilon} / \tau_{e}}}{1+k}-\frac{1+\psi}{(1-\alpha) \psi+(1+\alpha \psi) \eta_{c}} \alpha \eta_{c}>0,
$$

since $\frac{1+\psi}{(1-\alpha) \psi+(1+\alpha \psi) \eta_{c}} \alpha \eta_{c}<1$. If $\eta_{c} \geq \alpha$, then, since $\frac{\hat{\tau}_{A}^{c}}{\hat{\tau}_{A}} \leq 1$ and $\frac{1+\psi}{\psi+\alpha}>1$, so that $\frac{\lambda-\alpha}{\lambda-\alpha \frac{1+\psi}{\psi+\alpha}} \frac{1+\psi}{\psi+\alpha}>$ 1, we can bound $\frac{d \log L H S}{d A^{*}}$ from below by

$$
\begin{aligned}
\frac{d \log L H S}{d A^{*}} & \geq 1+\frac{\alpha}{1-\alpha} \frac{1+\psi}{\psi}+\frac{\sqrt{\tau_{\varepsilon} / \tau_{e}}}{1+k}-\frac{1}{1-\alpha} \frac{\psi+\alpha}{\psi} \frac{1+\psi}{(1-\alpha) \psi+(1+\alpha \psi) \eta_{c}} \eta_{c} \\
& =\frac{(\psi+\alpha)\left(1-\eta_{c}\right)}{(1-\alpha) \psi+(1+\alpha \psi) \eta_{c}}+\frac{\sqrt{\tau_{\varepsilon} / \tau_{e}}}{1+k}>0 .
\end{aligned}
$$

Consequently, $\frac{d \log L H S}{d A^{*}} \geq 0$, and therefore the LHS of equation (26) is (weakly) monotonically increasing in $A^{*}$. Since the LHS of equation (26) is monotonically increasing in $A^{*}$, while the RHS is fixed, it follows that the cutoff equilibrium is unique. Therefore, there exists a unique cutoff equilibrium with informational frictions.

Since $\hat{A}^{c}$ and $\hat{A}_{i}^{*}$ are both increasing in the public signal $Q$, it follows by applying the Implicit Function Theorem to equation (26) that

$$
\frac{d A^{*}}{d \varepsilon_{Q}}<0
$$

where $\varepsilon_{Q}$ is the noise in $Q$, since the LHS of equation (26) is nonnegative and (weakly) monotonically increasing in $A^{*}$. Since the noise in the public signal is independent of $A$, it follows that $\frac{d s}{d \varepsilon_{Q}}>0$, and more households enter the neighborhood in response to a more positive noise shock. Similarly, it also follows that $\frac{d P}{d \varepsilon_{Q}}>0$, and the housing price increases in response to the stronger housing demand.

By applying the Implicit Function Theorem to equation (26) with respect $z$, we see that

$$
\frac{d A^{*}}{d z}=\frac{\frac{1}{1+k} \sqrt{\frac{\tau_{\varepsilon}}{\tau_{e}}}-\frac{G^{\prime}\left(\hat{A}^{*}-A^{*}, \hat{\tau}_{A}\right)}{G\left(\hat{A}^{*}-A^{*}, \hat{\tau}_{A}\right)} \frac{\frac{\tau_{\varepsilon}}{\tau_{e}} \tau_{\xi}}{\tau_{A}+\tau_{Q}+\frac{\tau_{\varepsilon}}{\tau_{e}} \tau_{\xi}+\tau_{\varepsilon}}-\frac{\alpha \frac{1+\psi}{\psi+\alpha}}{\lambda-\alpha \frac{1+\psi}{\psi+\alpha}} \frac{F^{\prime}\left(\hat{A}^{c}-A^{*}, \hat{\tau}_{A}^{c}\right)}{F\left(\hat{A}^{c}-A^{*}, \hat{\tau}_{A}^{c}\right)} \frac{\frac{\tau_{\varepsilon}}{\tau_{e}} \tau_{\xi}}{\tau_{A}+\tau_{Q}+\frac{\tau_{\varepsilon}}{\tau_{e}} \tau_{\xi}}}{\frac{d \log L H S}{d A^{*}}} .
$$


Since $\frac{G^{\prime}\left(\hat{A}^{*}-A^{*}, \hat{\tau}_{A}\right)}{G\left(\hat{A}^{*}-A^{*}, \hat{\tau}_{A}\right)}, \frac{F^{\prime}\left(\hat{A}^{c}-A^{*}, \hat{\tau}_{A}^{c}\right)}{F\left(\hat{A}^{c}-A^{*}, \hat{\tau}_{A}^{c}\right)}>0$, we can find a sufficient condition for the learning effect to dominate the cost effect by truncating the $\frac{G^{\prime}\left(\hat{A}^{*}-A^{*}, \hat{\tau}_{A}\right)}{G\left(\hat{A}^{*}-A^{*}, \hat{\tau}_{A}\right)}$ term and recognizing $\frac{F^{\prime}\left(\hat{A}^{c}-A^{*}, \hat{\tau}_{A}^{c}\right)}{F\left(\hat{A}^{c}-A^{*}, \hat{\tau}_{A}^{c}\right)} \geq \alpha \frac{1+\psi}{\psi+\alpha+(1-\alpha) \eta_{c}}\left(1-\eta_{c}\right)$, since $\frac{1}{f(z)} \frac{d f(z)}{f(z)}$ achieves its minimum at this value. It then suffices for the learning effect to dominate the cost effect that

$$
\frac{1}{1+k} \sqrt{\frac{\tau_{\varepsilon}}{\tau_{e}}}-\frac{\alpha \frac{1+\psi}{\psi+\alpha}}{\lambda-\alpha \frac{1+\psi}{\psi+\alpha}} \alpha \frac{1+\psi}{\psi+\alpha+(1-\alpha) \eta_{c}}\left(1-\eta_{c}\right) \frac{\frac{\tau_{\varepsilon}}{\tau_{e}} \tau_{\xi}}{\tau_{A}+\tau_{Q}+\frac{\tau_{\varepsilon}}{\tau_{e}} \tau_{\xi}} \leq 0
$$

from which follows that it is sufficient, although not necessary, that

$$
\frac{1+k}{1+\frac{\tau_{e}}{\tau_{\varepsilon} \tau_{\zeta}}\left(\tau_{A}+\tau_{Q}\right) k} \geq \frac{\lambda-\alpha \frac{1+\psi}{\psi+\alpha}}{\alpha \frac{1+\psi}{\psi+\alpha}} \frac{\psi+\alpha+(1-\alpha) \eta_{c}}{\alpha\left(1-\eta_{c}\right)(1+\psi)} \sqrt{\frac{\tau_{\varepsilon}}{\tau_{e}}},
$$

for $\frac{d A^{*}}{d z}<0$. As a consequence, more households enter in response to the information in a higher housing price, and this impact is in(de)creasing in $k$ if $\frac{\tau_{e}}{\tau_{\varepsilon} \tau_{\zeta}}\left(\tau_{A}+\tau_{Q}\right) \leq(>) 1$. It then follows that, in addition, $\frac{d P}{d z}>0$ and more households also enter the neighborhood.

Finally, as $\tau_{Q} \nearrow \infty$, that $\hat{A}^{c}, \hat{A}^{i} \rightarrow$ a.s. $A$, since $\hat{\tau}_{A}^{c}, \hat{\tau}_{A}^{i} \nearrow \infty$. Taking the limit along a sequence of $\tau_{Q}$, equation (25) converges to equation (23), and therefore $A^{*}$ converges to its perfect-information benchmark value, as do the optimal labor and capital supply. Therefore the noisy rational expectations cutoff equilibrium converges to the perfect-information benchmark economy as $\tau_{Q} \nearrow \infty$.

\section{A.5 Proof of Proposition 5}

We begin with our analysis of the equilibrium at $t=2$, after informational frictions have dissipated after an arbitrary profile of housing policies by households. To see that this is the unique equilibrium in the economy, define the operator $T: \mathcal{B}^{\phi}(\mathbb{R}) \rightarrow \mathcal{B}^{\phi}(\mathbb{R})$ characterizing the optimal household $i$ 's optimal labor choice:

$$
\begin{aligned}
T(x(i))= & \frac{(1+\alpha \psi)\left(1-\eta_{c}\right)}{(1-\alpha) \psi+(1+\alpha \psi) \eta_{c}} A_{i}-\frac{(1+\alpha \psi) \alpha}{(1-\alpha) \psi+(1+\alpha \psi) \eta_{c}}(\log \alpha(1-\alpha)+\log R) \\
& +\frac{1+\alpha \psi}{(1-\alpha) \psi+(1+\alpha \psi) \eta_{c}} \log (1-\alpha)-\frac{(1+\alpha \psi) \eta_{c}}{(1-\alpha) \psi+(1+\alpha \psi) \eta_{c}} \log E\left[1_{\left\{H_{j}=1\right\}}\right] \\
& +\frac{(1+\alpha \psi) \eta_{c}}{(1-\alpha) \psi+(1+\alpha \psi) \eta_{c}} \log E\left[e^{A_{j}+x(j)} 1_{\left\{H_{j}=1\right\}}\right]
\end{aligned}
$$

where $\mathcal{B}^{\phi}(\mathbb{R})$ is the space of functions $\phi$-bounded in the $\phi$-norm $\|f\|_{\phi}=\sup _{z} \frac{|f(z)|}{\phi(z)}$ for $\phi(z)>0$. When households follow a cutoff strategy, then $E\left[1_{\left\{H_{j}=1\right\}}\right]=\Phi\left(\sqrt{\tau_{\varepsilon}}\left(A-A^{*}\right)\right)$ and $E\left[e^{A_{j}+x(j)} 1_{\left\{H_{j}=1\right\}}\right]=E\left[e^{A_{j}+x(j)} 1_{\left\{A_{j} \geq A^{*}\right\}}\right]$. We introduce the weighted norm since $x(i)$ is potentially unbounded. $T(x(i))$ is continuous across $i$, since the expectation operator is 
bounded and preserves continuity for lognormal $A_{j}$. Furthermore, $T(\cdot)$ satisfies monotonicity $T(y(i)) \geq T(x(i))$ whenever $y(i) \geq x(i)(\forall i)$, and discounting since

$$
T(x(i)+\beta)=T(x(i))+\frac{(1+\alpha \psi) \eta_{c}}{(1-\alpha) \psi+(1+\alpha \psi) \eta_{c}} \beta<T(x(i))+\beta \phi\left(x^{*}\right),
$$

for $x^{*}=\arg \sup \|f\|_{\phi(\cdot)}$ and a constant $\beta>0 . T$ is therefore a strict contraction map by the Weighted Contraction Mapping Theorem of Boyd (1990). Since a contraction map has, at most, one fixed point, if an equilibrium with a continuous $x(i)$ exists, it is the unique equilibrium, at least within the class of functions bounded in the $\phi-$ sup norm. Notice now that the choice of $\phi(\cdot)$ is arbitrary, as it does not impact the contractive properties of $T(\cdot) .^{21}$ We therefore conclude the $x(i)$ that solves the fixed-point equation is unique.

Since $x(i)=(1+\alpha \psi) l_{i}$ is unique in the economy, it follows that the function for capital $K_{i}$ is also unique. As such, total capital demand in the economy is unique, and the marketclearing rental rate $R$ is therefore also unique. Consequently, the equilibrium we derived is the unique equilibrium at $t=2$ in the economy, given the household decision strategy at $t=1,\left\{H_{i}\right\}_{i \in[0,1]}$.

In addition, we recognize from the functional fixed-point equation (27) that $l_{i}$ is strictly increasing in $A_{i}$, since one can take a sequence $l_{i}^{k}=T l_{i}^{k-1}$, for which $l_{i}^{k}$ is strictly increasing in $A_{i}$ along the sequence, and take the limit as $k \rightarrow \infty$. Furthermore, $l_{i}$ conditional on $A_{i}$ is increasing in $A$ from the functional fixed-point equation (27) by similar arguments, since $l_{i}$ is strictly increasing in $e^{A_{j}}=e^{A+\varepsilon_{j}}$ for any arbitrary housing policy.

We now turn our attention to $t=1$. Consider the problem of household $i$ when all other households follow arbitrary strategy profiles. Solving for the household $i^{\prime} s$ optimal consumption and production decisions at $t=2$, it follows we can express $K_{i}$ and $p_{i}$ as

$$
\begin{aligned}
K_{i} & =\frac{1}{\alpha(1-\alpha) R} l_{i}^{1+\psi}, \\
p_{i} & =\left(e^{A_{i}} l_{i}^{1+\alpha \psi}\right)^{-\eta_{c}}\left(E\left[1_{\left\{H_{j}=1\right\}}\right]^{-1} \int_{\mathcal{N} / i} e^{A_{j}} l_{j}^{1+\alpha \psi} d j\right)^{\eta_{c}},
\end{aligned}
$$

and, by imposing market-clearing in the market for capital, the price of capital is given by

$$
R=\frac{1}{\alpha(1-\alpha)} \frac{1}{E\left[1_{\left\{H_{j}=1\right\}}\right]} \frac{1}{K} \int_{\mathcal{N}} l_{j}^{1+\psi} d j
$$

Since household $i$ is atomistic, it follows, by substituting for $p_{i}, K_{i}$, and $R$, that

$$
E\left[U_{i} \mid \mathcal{I}_{i}\right]=(1-\alpha) \frac{\psi}{1+\psi} E\left[\left(e^{A_{i}} l_{i}^{1+\psi \alpha}\right)^{1-\eta_{c}} E\left[e^{A_{j}} l_{j}^{1+\psi \alpha} \mid j \in \mathcal{N}\right]^{\eta_{c}} E\left[l_{j}^{1+\psi} \mid j \in \mathcal{N}\right]^{-\alpha} K^{\alpha}|| \mathcal{I}_{i}\right] .
$$

\footnotetext{
${ }^{21}$ The choice of $\phi(\cdot)$ is not entirely without loss, as existence depends on the space of $\phi$-bounded functions being a complete metric space.
} 
Now fix $K$ as a parameter, since it is publicly observable to all households. Note that the term $e^{A_{i}} l_{i}^{1+\psi \alpha}$ is increasing in $A_{i}$, ignoring indirect effects through inference about $A$, and in $A$ conditional on $A_{i}$, since $l_{i}$ is increasing in these arguments. Now suppose that $A$ increases to $(1+\varepsilon) A$, holding fixed $A_{i}, P$, and $K$, and this increases $l_{j}$ to $(1+\delta) l_{j}$ for all $j$. Then

$$
\frac{\Delta E\left[U_{i} \mid \mathcal{I}_{i}\right]}{E\left[U_{i} \mid \mathcal{I}_{i}\right]}=(1+\varepsilon)(1+\delta)^{1-\alpha}>0
$$

and $E\left[U_{i} \mid \mathcal{I}_{i}\right]$ is also increasing in $A .{ }^{22}$ As all households share a common posterior about $A$ after observing the housing price, their private beliefs about $A$ and their private type $A_{j}$ are perfectly positively correlated. Consequently, we can express the expected utility of household $i$ as

$$
E\left[U_{i} \mid \mathcal{I}_{i}\right]=h\left(A_{i}, P, Q\right) K^{\alpha}
$$

with $\frac{\partial h}{\partial A_{i}}>0$ since the argument in the expectation is increasing in $A_{i}$ and $A$ realization-byrealization.

It then follows that household $i$ will follow a cutoff strategy, and buy if

$$
A_{i} \geq h^{-1}\left(P / K^{\alpha}, P, Q\right)
$$

with the cutoff determined by the participation of other households in the neighborhood. This confirms the optimality of their cutoff strategy in their private type. As this holds for any $P$ and $K$, the result follows for any $P$ and $K$.

In the special case of perfect information, we can express $E\left[U_{i} \mid \mathcal{I}_{i}\right]=(1-\alpha) \frac{\psi}{1+\psi} f\left(A_{i}\right) g(\mathcal{N})$, with $f\left(A_{i}\right)=\left(e^{A_{i}} l_{i}^{1+\psi \alpha}\right)^{1-\eta_{c}}$ is strictly increasing in $A_{i}$, while $g(\mathcal{N})$ is independent of $A_{i}$. Household $i$ will then follow a cutoff strategy, and buy if

$$
A_{i} \geq f^{-1}(P / g(\mathcal{N}))
$$

with the cutoff determined by the participation of other households in the neighborhood.

Furthermore, we recognize that builders, regardless of their beliefs about demand fundamental, $A$, will follow cutoff strategies when choosing whether to supply a house. By market-clearing and rational expectations, the functional form for the housing price and the equilibrium beliefs of households follow.

Given that the housing price has the conjectured functional form, capital producers form rational expectations about $A$, and their optimal supply of capital is unique from the concavity of their optimization program. As such, the cutoff equilibrium we characterized is the unique rational expectations equilibrium in the economy, both with informational frictions and perfect-information.

\footnotetext{
${ }^{22}$ In the background, the utility of households, conditional on $A$, is supermodular in $A_{i}$ and the actions of the other households.
} 